\title{
Deleting and Inserting Fixed Point Manifolds under the Weak Gap Condition
}

\author{
Dedicated to Professor Krzysztof Pawałowski on his 60th birthday
}

by

Masaharu Morimoto

\begin{abstract}
Let $G$ be a finite group and $X$ a compact smooth manifold. It is of interest which smooth manifolds can be the $G$-fixed point sets of smooth $G$-actions on $X$. The deleting-inserting theorem of this paper is related to this problem and has applications to one-fixed-point actions on spheres as well as to Smith equivalence.
\end{abstract}

2010 Mathematics Subject Classification: Primary 57S17; Secondary 57R67, 19J25, 20 C05. Keywords: equivariant surgery, surgery obstruction, weak gap condition, homology equivalence, Smith equivalence.

\section{§1. Introduction}

Let $G$ be a finite group. In this paper, a manifold and a $G$-manifold mean a smooth manifold and a smooth $G$-manifold, respectively. Given a manifold $X$, it is a fundamental problem to study which manifolds and real vector bundles can be the $G$-fixed point sets and the normal bundles of $G$-fixed point sets, respectively, of smooth $G$-actions on $X$. This problem for the case where $X$ is a disk was studied by B. Oliver [15], and for $X$ a sphere in [11] under the gap condition. The Smith problem on tangential representations at fixed points on spheres is a part of the problem above and has been studied by various authors. It has been useful for the study of the problem to delete (or insert) manifolds from (or to) a given manifold $X$ as $G$-fixed point sets. More precisely, for a given $G$-manifold $Y$ having the diffeomorphism type of $X$ and the $G$-fixed point set

$$
Y^{G}=F_{1} \amalg \cdots \amalg F_{m}
$$

Communicated by T. Ohtsuki. Received September 15, 2011. Revised November 20, 2011.

M. Morimoto: Department of Mathematics, Graduate School of Natural Science and Technology, Okayama University, 3-1-1 Tsushimanaka, Kitaku, Okayama, 700-8530 Japan;

e-mail: morimoto@ems . okayama-u.ac.jp 
and given integers $1 \leq r_{1} \leq \cdots \leq r_{n} \leq m$, it is of interest whether there exists a $G$-manifold $Z$ having the diffeomorphism type of $X$ and the $G$-fixed point set

$$
Z^{G}=F_{r_{1}} \amalg \cdots \amalg F_{r_{n}} .
$$

A finite group $G$ is called an Oliver group if there exists a smooth $G$-action on a disk without $G$-fixed points, or equivalently if there never exists a normal series $P \unlhd H \unlhd G$ such that $P$ and $G / H$ have prime power order and $H / P$ is a cyclic group (cf. $[16,15,6]$ ). We studied such deleting-inserting methods for an Oliver group $G$ invoking the gap condition for which the main requirement is

$$
2 \operatorname{dim} Y^{g}<\operatorname{dim} Y
$$

for all non-trivial elements $g$ of $G$, i.e. $g \neq e$. In the current paper we give a deleting-inserting theorem (Theorem 5.1) for an Oliver group under the weak gap condition which allows the case that $2 \operatorname{dim} Y^{g}=\operatorname{dim} Y$ for $g \in G$. This theorem yields Theorems $1.3-1.10$ below as applications.

Let $\mathcal{S}(G)$ denote the set of all subgroups of $G$, and $\mathcal{P}(G)$ the set of all primepower-order subgroups of $G$, where by convention $\{e\} \in \mathcal{P}(G)$. For a prime $p$, let $G^{\{p\}}$ denote the smallest normal subgroup $N$ of $G$ such that $|G / N|$ is a power of $p$, possibly $|G / N|=1$. Let $\mathcal{L}(G)$ denote the set of all subgroups $H$ containing $G^{\{p\}}$ for some prime $p$. A (finite-dimensional) real $G$-module $V$ is called $\mathcal{L}$-free if $V^{L}=0$ for all $L \in \mathcal{L}(G)$. We define a $G$-submodule $V_{\mathcal{L}}$ of $V$ by

$$
V_{\mathcal{L}}=\left(V-V^{G}\right)-\bigoplus_{p \text { prime }}\left(V^{G^{\{p\}}}-V^{G}\right) .
$$

Let $\mathbb{R}[G]$ denote the group ring of $G$ with real coefficients having the canonical (left) $G$-action. Recall the following fact.

Lemma 1.1 ([6, Theorem 2.3]). The real $G$-module $V=\mathbb{R}[G]_{\mathcal{L}}$ has the following properties:

(1.1.1) $V^{H}=0$ if and only if $H \in \mathcal{L}(G)$.

(1.1.2) $\operatorname{dim} V^{H} \geq|K: H| \operatorname{dim} V^{K}$ for all $H \leq K \in \mathcal{S}(G)$.

(1.1.3) The equality $\operatorname{dim} V^{H}=2 \operatorname{dim} V^{K}$ holds, where $H \leq K \in \mathcal{S}(G)$, if and only if $|K: H|=2,\left|K G^{\{2\}}: H G^{\{2\}}\right|=2$, and $H G^{\{q\}}=G$ for all odd primes $q$.

By straightforward computation, we can show the next lemma.

Lemma $1.2\left(\left[13\right.\right.$, Proposition 1.9]). If $G$ is an Oliver group then $\operatorname{dim}\left(\mathbb{R}[G]_{\mathcal{L}}\right)^{P}$ $\geq 2$ for all $P \in \mathcal{P}(G)$. 
The following two theorems are an elaboration of [6, Theorem B]. In particular, for $m=1$ they give smooth one-fixed-point actions on spheres.

Theorem 1.3. Let $G$ be an Oliver group and $m$ a positive integer. Then for any integer $\ell \geq 3$ there exists a $G$-action on the standard sphere $S$ of dimension

$$
d_{\ell}=\ell \cdot\left\{(|G|-1)-\sum_{p \||G|}\left(\left|G / G^{\{p\}}\right|-1\right)\right\}
$$

with exactly $m$ G-fixed points $x_{1}, \ldots, x_{m}$ for which the tangential representations $T_{x_{i}}(S)$ are all isomorphic to the $\ell$-fold direct sum $\mathbb{R}[G]_{\mathcal{L}}^{\oplus \ell}$ of $\mathbb{R}[G]_{\mathcal{L}}$.

Let $\mathcal{P H}(G)$ denote the set of all pairs $(P, H)$ consisting of $P \in \mathcal{P}(G)$ and $H \in \mathcal{S}(G)$ with $P<H$. Let $\mathcal{P H}_{2}(G)$ denote the set of all pairs $(P, H) \in \mathcal{P H}(G)$ such that $|H: P|=2,\left|H G^{\{2\}}: P G^{\{2\}}\right|=2$, and $P G^{\{q\}}=G$ for all odd primes $q$. For a set $\mathcal{A}$ of pairs $(H, K)$ with $H<K \in \mathcal{S}(G)$, we say that a real $G$-module $V$ satisfies the gap condition (resp. the weak gap condition) on $\mathcal{A}$ if

$$
\operatorname{dim} V^{H}>2 \operatorname{dim} V^{K} \quad\left(\text { resp. } \operatorname{dim} V^{H} \geq 2 \operatorname{dim} V^{K}\right)
$$

for any $(H, K) \in \mathcal{A}$. It should be remarked that if an $\mathcal{L}$-free real $G$-module $V$ satisfies the weak gap condition on $\mathcal{P H}_{2}(G)$ then $V \oplus \mathbb{R}[G]_{\mathcal{L}}^{\oplus m}$ satisfies the weak gap condition on $\mathcal{P H}(G)$ for any $m \geq \operatorname{dim} V$.

Theorem 1.4. Let $G$ be an Oliver group, $m$ a positive integer, and $V$ an $\mathcal{L}$-free real $G$-module satisfying the weak gap condition on $\mathcal{P H}_{2}(G)$. Then there exists an integer $N$ such that for every integer $\ell \geq N$ there exists a $G$-action on the standard sphere $S$ with exactly $m G$-fixed points $x_{1}, \ldots, x_{m}$ for which the tangential representations $T_{x_{i}}(S)$ are all isomorphic to $V \oplus \mathbb{R}[G]_{\mathcal{L}}^{\oplus \ell}$.

Let $\operatorname{RO}(G)$ denote the real representation ring. For a subset $A$ of $\operatorname{RO}(G), A_{\mathcal{P}}$ stands for the set

$$
A \cap \bigcap_{P \in \mathcal{P}(G)} \operatorname{Ker}\left[\operatorname{res}_{P}^{G}: \operatorname{RO}(G) \rightarrow \operatorname{RO}(P)\right]
$$

Real $G$-modules $V$ and $W$ are called Smith equivalent if there exists a homotopy sphere $\Sigma$ with a $G$-action such that $\Sigma^{G}$ consists of exactly two points $a$ and $b$, and the tangential representations $T_{a}(\Sigma)$ and $T_{b}(\Sigma)$ are isomorphic to $V$ and $W$, respectively. Let $\operatorname{Sm}(G)$ denote the Smith set of $G$, i.e.

$$
\operatorname{Sm}(G)=\{[V]-[W] \in \operatorname{RO}(G) \mid V \text { is Smith equivalent to } W\} .
$$


The subset $\operatorname{Sm}(G)_{\mathcal{P}}$ is called the primary Smith set of $G$. For a subset $A$ of $\operatorname{RO}(G)$, $A^{\mathcal{L}}$ stands for the set

$$
\left\{[V]-[W] \in A \mid V^{L}=0 \text { and } W^{L}=0 \text { for all } L \in \mathcal{L}(G)\right\} .
$$

We say that two real $G$-modules $V$ and $W$ are $\mathcal{P}$-matched if $\operatorname{res}_{P}^{G} V$ and $\operatorname{res}_{P}^{G} W$ are isomorphic for all $P \in \mathcal{P}(G)$.

Theorem 1.5. Let $G$ be an Oliver group. Let $V_{1}, \ldots, V_{m}$ be $\mathcal{L}$-free real $G$-modules satisfying the weak gap condition on $\mathcal{P} \mathcal{H}_{2}(G)$, of which arbitrary two are $\mathcal{P}$-matched. Then there exists an integer $N$ such that for any integer $\ell \geq N$, there exists a smooth $G$-action on the standard sphere $S$ with exactly $m G$-fixed points $x_{1}, \ldots, x_{m}$ for which the tangential representation $T_{x_{i}}(S)$ is isomorphic to $V_{i} \oplus \mathbb{R}[G]_{\mathcal{L}}^{\oplus \ell}, 1 \leq$ $i \leq m$.

In the case $m=2$, we obtain the next theorem on Smith equivalence.

Theorem 1.6. Let $G$ be an Oliver group and let $V$ and $W$ be $\mathcal{P}$-matched and $\mathcal{L}$ free real $G$-modules both satisfying the weak gap condition on $\mathcal{P} \mathcal{H}_{2}(G)$. Then there exists an integer $N$ such that for any integer $\ell \geq N$ there exists a smooth $G$-action on the standard sphere $S$ with exactly two $G$-fixed points $x_{1}$ and $x_{2}$ for which the tangential representations $T_{x_{1}}(S)$ and $T_{x_{2}}(S)$ are isomorphic to $V \oplus \mathbb{R}[G]_{\mathcal{L}}^{\oplus \ell}$ and $W \oplus \mathbb{R}[G]_{\mathcal{L}}^{\oplus \ell}$, respectively. In particular, $V$ and $W$ are stably Smith equivalent.

Let $X$ be a $G$-manifold and $S$ a smooth $G$-action on the standard sphere with exactly one $G$-fixed point $a$ and $T_{a}(S) \cong \mathbb{R}[G]_{\mathcal{L}}^{\oplus \ell}$. Then the cartesian product $Y=$ $X \times S$ has the diagonal $G$-action and the $G$-fixed point set of $Y$ is $X^{G} \times\{a\}$. For each $x \in X^{G}$, the tangential representation $T_{(x, a)}(Y)$ is isomorphic to $T_{x}(X) \oplus \mathbb{R}[G]_{\mathcal{L}}^{\oplus \ell}$. The next theorem follows from Theorems 1.3 and 1.6.

Theorem 1.7. Let $G$ be an Oliver group and $\left(V_{i}, W_{i}\right)$ a pair of $\mathcal{L}$-free $\mathcal{P}$-matched real $G$-modules $V_{i}$ and $W_{i}$ for each $1 \leq i \leq m$. Suppose all $V_{i}$ and $W_{i}, 1 \leq i \leq m$, satisfy the weak gap condition on $\mathcal{P H}_{2}(G)$. Let $X$ be a $G$-manifold with $G$-fixed point set

$$
X^{G}=\left\{x_{1}\right\} \amalg \cdots \amalg\left\{x_{m}\right\} \amalg F \quad \text { (disjoint union) }
$$

such that for each $1 \leq i \leq m$, the tangential representation $T_{x_{i}}(X)$ is isomorphic to $V_{i}$, where $F$ is a union of connected components of $X^{G}$. Then there exists an integer $N$ such that for any integer $\ell \geq N$ there exists a $G$-manifold $Y$ with $G$-fixed point set $X^{G}$ for which the underlying space is diffeomorphic to $X \times S\left(\mathbb{R} \oplus \mathbb{R}[G]_{\mathcal{L}}^{\oplus \ell}\right)$ and the tangential representation $T_{x_{i}}(Y)$ is isomorphic to $W_{i} \oplus \mathbb{R}[G]_{\mathcal{L}}^{\oplus \ell}$ for each $1 \leq i \leq m$. 
A finite group $G$ is called a gap group if each element $x$ of $\operatorname{RO}(G)_{\mathcal{P}}^{\mathcal{L}}$ can be written in the form $x=[V]-[W]$ with $\mathcal{L}$-free real $G$-modules $V$ and $W$ satisfying the gap condition on $\mathcal{P} \mathcal{H}(G)$. We remark that $G$ with $\mathcal{L}(G) \cap \mathcal{P}(G)=\emptyset$ is a gap group if and only if there exists an $\mathcal{L}$-free real $G$-module $V$ satisfying the gap condition on $\mathcal{P H}_{2}(G)$. An Oliver group $G$ is a gap group if $G$ is nilpotent, or $G=G^{\{2\}}$, or $G \neq G^{\{p\}}$ for at least two odd primes $p$. In the case where $G$ is a gap Oliver group, we could determine the geometrically defined set $\operatorname{Sm}(G)_{\mathcal{P}} \mathcal{L}$ in algebraic terms: $\operatorname{Sm}(G)_{\mathcal{P}}^{\mathcal{L}}$ coincides with $\operatorname{RO}(G)_{\mathcal{P}}^{\mathcal{L}}$ (cf. [17, p. 850, Realization Theorem]). But it is difficult to determine $\operatorname{Sm}(G)$ or even $\operatorname{Sm}(G)_{\mathcal{P}}^{\mathcal{L}}$ when $G$ is not a gap group. Let us call a finite group $G$ a weak gap group if each element $x$ of $\operatorname{RO}(G)_{\mathcal{P}}^{\mathcal{L}}$ can be written in the form $x=[V]-[W]$ with $\mathcal{L}$-free real $G$-modules $V$ and $W$ satisfying the weak gap condition on $\mathcal{P H}(G)$. For example, $G=S_{5} \times C_{2} \times \cdots \times C_{2}$ is not a gap group but a weak gap group (cf. [4]), where $S_{5}$ is the symmetric group on five letters and $C_{2}$ is a group of order 2. Since $\operatorname{Sm}(G)_{\mathcal{P}}^{\mathcal{L}} \subset \operatorname{RO}(G)_{\mathcal{P}}^{\mathcal{L}}$, we obtain the next result.

Theorem 1.8. If $G$ is a weak gap Oliver group then $\operatorname{Sm}(G)_{\mathcal{P}}^{\mathcal{L}}$ coincides with $\operatorname{RO}(G)_{\mathcal{P}}^{\mathcal{L}}$.

Let $H$ be a subgroup of $G$. For a real $H$-module $V$, we denote by $\operatorname{ind}_{H}^{G} V$ the real $G$-module $\mathbb{R}[G] \otimes_{\mathbb{R}[H]} V$. If $V$ satisfies the weak gap condition on $\mathcal{P} \mathcal{H}(H)$ then $\operatorname{ind}_{H}^{G} V$ satisfies the weak gap condition on $\mathcal{P} \mathcal{H}(G)$; if $V$ is $\mathcal{L}$-free then $\operatorname{ind}_{H}^{G} V$ is also $\mathcal{L}$-free; and if $V$ and $W$ are $\mathcal{P}$-matched real $H$-modules then $\operatorname{ind}_{H}^{G} V$ and $\operatorname{ind}_{H}^{G} W$ are $\mathcal{P}$-matched real $G$-modules. Let ind ${ }_{H}^{G}$ denote the induction homomorphism $\mathrm{RO}(H) \rightarrow \mathrm{RO}(G)$. Then the inclusion $\operatorname{ind}_{H}^{G}\left(\mathrm{RO}(H)_{\mathcal{P}}^{\mathcal{L}}\right) \subset \operatorname{RO}(G)_{\mathcal{P}}^{\mathcal{L}}$ holds. Thus we obtain the next result from Theorem 1.6.

Theorem 1.9. Let $H$ be a subgroup of an Oliver group $G$.

(1.9.1) If $V$ and $W$ are $\mathcal{L}$-free $\mathcal{P}$-matched real $H$-modules satisfying the weak gap condition on $\mathcal{P} \mathcal{H}_{2}(H)$ then $\left[\operatorname{ind}_{H}^{G} V\right]-\left[\operatorname{ind}_{H}^{G} W\right]$ belongs to $\operatorname{Sm}(G)_{\mathcal{P}}^{\mathcal{L}}$.

(1.9.2) If $H$ is a weak gap group then

$$
\operatorname{ind}_{H}^{G}\left(\operatorname{Sm}(H)_{\mathcal{P}}^{\mathcal{L}}\right) \subset \operatorname{ind}_{H}^{G}\left(\operatorname{RO}(H)_{\mathcal{P}}^{\mathcal{L}}\right) \subset \operatorname{Sm}(G)_{\mathcal{P}}^{\mathcal{L}} .
$$

Let $\mathcal{H}(G)$ denote the set of all subgroups $H$ of $G$ for which there exists $P \in$ $\mathcal{P}(G)$ such that $P \leq H$ and $|H: P| \leq 2$. For a subset $A \subset \operatorname{RO}(G)$, we define $A_{\mathcal{H}}$ to be the set of all elements $x \in A$ such that $\operatorname{res}_{H}^{G} x=0$ for all $H \in \mathcal{H}(G)$. It is obvious that $A_{\mathcal{H}}^{\mathcal{L}} \subset \operatorname{RO}(G)_{\mathcal{P}}^{\mathcal{L}}$.

Theorem 1.10. If $H$ is a subgroup of an Oliver group $G$ then

$$
\operatorname{ind}_{H}^{G}\left(\operatorname{RO}(H)_{\mathcal{H}}^{\mathcal{L}}\right) \subset \operatorname{Sm}(G)_{\mathcal{H}}^{\mathcal{L}} \subset \operatorname{Sm}(G)_{\mathcal{P}}^{\mathcal{L}} .
$$


This paper is organized as follows. Section 2 is devoted to preparation of basic terms and notation concerning $G$-manifolds and $G$-framed maps. In Section 3, we discuss equivariant surgery to obtain homology equivalences on even-dimensional manifolds satisfying the weak gap condition. Theorem 3.5 describes a surgery obstruction to $\mathbb{Z}_{(p)}$-homology equivalence in algebraic terms. Section 4 is devoted to the induction theory of equivariant surgery obstruction groups. In Section 5 we prove Theorem 5.1 which provides a method of deleting or inserting fixed point manifolds. Theorems $1.3-1.5$ and 1.10 are proved in Section 6 .

\section{§2. Preliminaries}

For families $\mathcal{A}, \mathcal{B}$ of sets closed under intersection, and a map $f: \mathcal{A} \rightarrow \mathcal{B}$, we say that $f$ preserves intersection or is intersection preserving if

$$
f\left(A_{1} \cap A_{2}\right)=f\left(A_{1}\right) \cap f\left(A_{2}\right) \quad \text { for all } A_{1}, A_{2} \in \mathcal{A} .
$$

Let $\Theta$ be a $G$-set, $\rho: \Theta \rightarrow \mathcal{S}(G)$ a $G$-map, where $G$ acts on $\mathcal{S}(G)$ by conjugation, and $S$ a conjugation invariant subset of $G$ consisting of elements of order 2 . The group $G$ acts on $S$ by conjugation. The set $\Theta$ is called $(\rho, S)$-simple if for each $t \in \Theta$, the set $\rho(t)$ contains at most one element in $S$.

Definition 2.1. For a $(\rho, S)$-simple $G$-set $\Theta$, we define the $S$-contraction $(\Theta / S, \rho / S)$ of $(\Theta, \rho)$ as follows. Let $\sim_{S}$ denote the equivalence relation on $\Theta$ such that $t \sim_{S} t^{\prime}$ if and only if $\rho(t) \cap S=\rho\left(t^{\prime}\right) \cap S$. Denote by $\Theta / S$ the set of equivalence classes with respect to $\sim_{S}$. The map $\rho / S: \Theta / S \rightarrow \mathcal{S}(G)$ is defined by

$$
\rho / S([t])=\{e\} \cup(\rho(t) \cap S)
$$

for the $\sim_{S}$-equivalence class $[t]$ of $t \in \Theta$. Then $\Theta / S$ has a canonical $G$-action and $\rho / S: \Theta / S \rightarrow \mathcal{S}(G)$ is a $G$-map.

A $G$-map $\rho: \Theta \rightarrow \mathcal{S}(G)$ is called $S$-injective (resp. $S$-bijective) if for each $s \in S$, there exists at most one (resp. exactly one) element $t \in \Theta$ such that $\rho(t)$ contains $s$.

Let $\mathfrak{P}(\Theta)$ denote the set of all subsets of $\Theta$. Clearly $\mathfrak{P}(\Theta)$ has the induced $G$-action. A $G$-map $f: \mathcal{S}(G) \rightarrow \mathfrak{P}(\Theta)$ is called $\rho$-compatible if $\rho(f(H)) \subset \mathcal{S}(H)$ for all $H \in \mathcal{S}(G)$. A $G$-map $f: \mathcal{S}(G) \rightarrow \mathfrak{P}(\Theta)$ is called $(\rho, S)$-saturated if

$$
f(H) \supset\{t \in \Theta \mid \rho(t) \cap S \cap H \neq \emptyset\} \quad \text { for all } H \in \mathcal{S}(G) .
$$

It is straightforward to verify the next lemma.

Lemma 2.2. Let $f: \mathcal{S}(G) \rightarrow \mathfrak{P}(\Theta)$ be an intersection preserving $\rho$-compatible $G$-map and set $\Theta_{H}=f(H)$ and $\rho_{H}=\left.\rho\right|_{\Theta_{H}}: \Theta_{H} \rightarrow \mathcal{S}(H)$. 
(2.2.1) If $\Theta$ is $(\rho, S)$-simple, then $\Theta_{H}$ is $\left(\rho_{H}, S \cap H\right)$-simple for $H \in \mathcal{S}(G)$ and the associated map $\rho / S: \Theta / S \rightarrow \mathcal{S}(G)$ is $S$-injective.

(2.2.2) If $\rho: \Theta \rightarrow \mathcal{S}(G)$ is $S$-injective then $\rho_{H}: \Theta_{H} \rightarrow \mathcal{S}(H)$ is $(S \cap H)$-injective for $H \in \mathcal{S}(G)$.

(2.2.3) If $\rho: \Theta \rightarrow \mathcal{S}(G)$ is $S$-bijective and $f: \mathcal{S}(G) \rightarrow \mathfrak{P}(\Theta)$ is $(\rho, S)$-saturated then $\rho_{H}: \Theta_{H} \rightarrow \mathcal{S}(H)$ is $(S \cap H)$-bijective for $H \in \mathcal{S}(G)$.

Let $X$ be a compact, connected $G$-manifold, possibly with boundary $\partial X$. The singular set $X_{\text {sing }}$ of $X$ is defined by

$$
X_{\text {sing }}=\bigcup_{g \in G \backslash\{e\}} X^{g} .
$$

We say that $X$ satisfies the weak gap condition if

$$
\operatorname{dim} X_{\text {sing }} \leq \frac{1}{2} \operatorname{dim} X .
$$

In the case where $X$ has even dimension $2 k$ and satisfies the weak gap condition, we say that $X$ satisfies the $k$-tame condition if

$$
\begin{gathered}
\operatorname{dim} X^{K} \leq k-2 \\
\text { whenever } H<K \in \mathcal{S}(G), \operatorname{dim} X^{H}=k, \text { and } H=\bigcap_{x \in X^{H}} G_{x},
\end{gathered}
$$

where $G_{x}$ stands for the isotropy subgroup of $G$ at the point $x$. Let $G(2)$ denote the set of all elements of $G$ of order 2. In the case where $X$ has even dimension $2 k$ and satisfies the weak gap condition, we say that $X$ satisfies the $G(2)$-condition if

$$
|H|=2 \quad \text { whenever } \quad H \in \mathcal{S}(G) \text { and } 2 \operatorname{dim} X^{H}=\operatorname{dim} X .
$$

For a subgroup $H$ and an integer $\ell \geq 0$, let $\pi_{0}\left(X^{H}, \ell\right)$ denote the set of all connected components of dimension $\ell$ of $X^{H}$. For $\alpha \in \pi_{0}\left(X^{H}, \ell\right)$, we denote by $X_{\alpha}$ or $X_{\alpha}^{H}$ the underlying space of $\alpha$. Each $\alpha \in \pi_{0}\left(X^{H}, \ell\right)$ determines the group

$$
\rho_{X}(\alpha)=\bigcap_{x \in X_{\alpha}} G_{x}
$$

Definition 2.3. Let $X$ be a compact, connected $G$-manifold, possibly with boundary, satisfying the weak gap condition. Then we set

$$
\begin{aligned}
& S(X)=\left\{g \in G \mid 2 \operatorname{dim} X^{g}=\operatorname{dim} X\right\}, \\
& Q(X)=\left\{g \in G \mid \operatorname{dim} X^{g}=[(\operatorname{dim} X-1) / 2]\right\}, \\
& \Sigma(X)=\left\{\alpha \mid H \in \mathcal{S}(G), \alpha \in \pi_{0}\left(X^{H}, \operatorname{dim} X / 2\right), \text { and } \rho_{X}(\alpha)=H\right\},
\end{aligned}
$$


where for a real number $x,[x]$ denotes the greatest integer not exceeding $x$. The $(\operatorname{dim} X / 2)$-dimensional singular structure $\mathfrak{S}(X)$ associated with $X$ is defined to be the set of all $X_{s}, s \in \Sigma(X)$. For each $s \in \Sigma(X)$, the manifold $X_{s}$ has the unique orientation class $t_{s}$ in $H_{k}\left(X_{s}, \partial X_{s} ; \mathbb{Z}_{2}\right)$. The $G$-set $\Theta^{(2)}(X)$ is defined to be the set of all $t_{s}$, where $s$ runs over $\Sigma(X)$. The correspondence $s \mapsto t_{s}$ gives a bijection $\Sigma(X) \rightarrow \Theta^{(2)}(X)$. The map $\rho_{X}^{(2)}: \Theta^{(2)}(X) \rightarrow \mathcal{S}(G)$ is defined by $\rho_{X}^{(2)}\left(t_{s}\right)=\rho_{X}(s)$ for $s \in \Sigma(X)$.

The proof of the next lemma is straightforward.

Lemma 2.4. Let $X$ be a $G$-manifold as in Definition 2.3. Suppose that $X$ has even dimension $n=2 k$ and satisfies the $G(2)$-condition. Then the following hold:

(2.4.1) $\Theta^{(2)}(X)$ is $\left(\rho_{X}^{(2)}, S(X)\right)$-simple.

(2.4.2) $\rho_{X}^{(2)} / S(X): \Theta^{(2)}(X) / S(X) \rightarrow \mathcal{S}(G)$ is $S(X)$-bijective.

(2.4.3) For $H \in \mathcal{S}(G), S\left(\operatorname{res}_{H}^{G} X\right)$ coincides with $S(X) \cap H$. Thus the map $H \mapsto$ $S\left(\operatorname{res}_{H}^{G} X\right)$ is intersection preserving.

(2.4.4) For $H \in \mathcal{S}(G), \Theta^{(2)}\left(\operatorname{res}_{H}^{G} X\right)$ coincides with $\left\{t \in \Theta^{(2)}(X) \mid \rho_{X}^{(2)}(t) \subset H\right\}$. Hence the map $f: \mathcal{S}(G) \rightarrow \mathfrak{P}\left(\Theta^{(2)}(X)\right) ; H \mapsto \Theta^{(2)}\left(\operatorname{res}_{H}^{G} X\right)$, is intersection preserving, $\rho_{X}^{(2)}$-compatible, and $\left(\rho_{X}^{(2)}, S(X)\right)$-saturated, and furthermore $f(G)=\Theta^{(2)}(X)$.

(2.4.5) The canonical map $\gamma: \Theta^{(2)}(X) \rightarrow \Theta^{(2)}(X) / S(X)$ is a $G$-map, the diagram

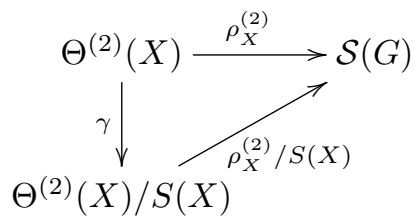

commutes, and

$$
\gamma\left(\Theta^{(2)}(X)\right)=\Theta^{(2)}(X) / S(X) .
$$

Let $X$ be a compact, connected, oriented $G$-manifold of dimension $n \geq 5$, possibly with boundary $\partial X$. Let $R$ be a commutative ring with 1 and with trivial anti-involution ${ }^{-}$. The group ring $R[G]$ has the anti-involution ${ }^{-}$derived from the orientation homomorphism $w_{X}: G \rightarrow\{ \pm 1\}$ of $X$, i.e.

$$
\left(\sum_{g \in G} r_{g} g\right)^{-}=\sum_{g \in G} r_{g} w_{X}(g) g^{-1},
$$

where $r_{g} \in R$. Let $\widetilde{X}$ denote the universal covering space of $X$. Let $\widetilde{G}$ denote the fundamental group $\pi_{1}\left(E G \times_{G} X\right)$, where $E G$ is a contractible $G$-CW complex with 
a free $G$-action. We have the exact sequence

$$
1 \rightarrow \pi_{1}(X) \rightarrow \widetilde{G} \rightarrow G \rightarrow 1
$$

If $X^{G}$ is nonempty then this sequence splits, i.e. $\widetilde{G}=\pi_{1}(X) \rtimes G$.

Let $Y$ be a compact, connected, oriented $G$-manifold of dimension $n$, possibly with boundary $\partial Y$. Let $f=(f, b)$ be a $G$-framed map, where $f:(X, \partial X) \rightarrow$ $(Y, \partial Y)$ is a $G$-map such that $f: X \rightarrow Y$ is 1 -connected, and $b: T(X) \oplus f^{*} \eta \rightarrow f^{*} \xi$ is a real $G$-vector bundle isomorphism for real $G$-vector bundles $\eta$ and $\xi$ over $Y$ such that $\eta \supset \varepsilon_{Y}\left(\mathbb{R}^{n}\right)$ (cf. [2, Lemma 6.1]). Then $f$ is covered by the induced $\widetilde{G}$-framed map $\tilde{\boldsymbol{f}}=(\widetilde{f}, \widetilde{b})$ consisting of a $\widetilde{\varphi}$-map $\tilde{f}:(\widetilde{X}, \partial \widetilde{X}) \rightarrow(\widetilde{Y}, \partial \widetilde{Y})$ and a real $\widetilde{G}$-vector bundle isomorphism $\widetilde{b}: T(\widetilde{X}) \oplus \tilde{f}^{*} \widetilde{\eta} \rightarrow \widetilde{f}^{*} \widetilde{\xi}$, where $\widetilde{Y}$ is the universal covering space of $Y, \widetilde{\varphi}$ is the canonical homomorphism $\widetilde{G}=\pi_{1}\left(E G \times_{G} X\right) \rightarrow$ $\pi_{1}\left(E G \times_{G} Y\right)=\widehat{G}$, and $\tilde{\eta}$ and $\widetilde{\xi}$ are the real $\widehat{G}$-vector bundles over $\widetilde{Y}$ induced from $\eta$ and $\xi$, respectively:

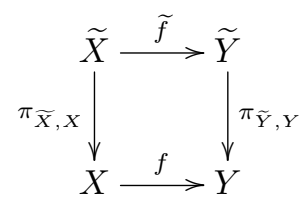

We note that the map $\tilde{f}:(\tilde{X}, \partial \widetilde{X}) \rightarrow(\widetilde{Y}, \partial \tilde{Y})$ is not necessarily of degree one.

\section{§3. G-surgery maps on even-dimensional manifolds}

Let $X$ be a compact, connected, oriented $G$-manifold of even dimension $n=$ $2 k \geq 6$, possibly with boundary $\partial X$. Throughout this section, we assume that $X$ satisfies the weak gap condition and the $k$-tame condition. Let $R$ be a commutative ring with 1 and with trivial anti-involution - . We set $\lambda=(-1)^{k}, S=S(X)$ and $Q=Q(X)$; further define

$$
(Q)_{R}=R[Q]+\{x-\lambda \bar{x} \mid x \in R[G]\}, \quad(S)_{R}=R[S]+\{x+\lambda \bar{x} \mid x \in R[G]\} .
$$

Then

$$
\boldsymbol{A}_{X}=\left(R[G],(\overline{\ulcorner}, \lambda),(S)_{R}, G, R[S],(Q)_{R}+R[S]\right)
$$

is a double parameter algebra in the sense of [2, Definition 2.5].

Let $\mathfrak{S}=\left\{X_{s} \mid s \in \Sigma\right\}$ be a set of compact connected $k$-dimensional neat submanifolds of $X$, where $\Sigma$ is a $G$-set, such that $g X_{s}=X_{g s}$ for all $g \in G$ and $s \in \Sigma$. Set

$$
X_{\mathfrak{S}}=\bigcup_{s \in \Sigma} X_{s} .
$$


In this paper, we assume that $\mathfrak{S}$ satisfies the $k$-tame condition, i.e.

$$
X_{s} \cap X_{t} \text { is a neat submanifold of } X_{s} \text { of dimension } \leq k-2
$$

for all $s, t \in \Sigma, s \neq t$. If $\mathfrak{S} \supset \mathfrak{S}(X)$ then we call $\mathfrak{S}$ a $k$-singular structure of $X$. The index set $\Sigma$ decomposes into the disjoint union of $\Sigma_{+}$and $\Sigma_{-}$consisting of all elements $s \in \Sigma$ such that $X_{s}$ is orientable and non-orientable, respectively. Let $\Theta^{(0)}(\mathfrak{S})$ denote the set of all generators of $H_{k}\left(X_{s}, \partial X_{s} ; \mathbb{Z}\right)$, where $s$ runs over $\Sigma_{+}$, and let $\Theta^{(2)}(\mathfrak{S})$ denote the set of all generators of $H_{k}\left(X_{s}, \partial X_{s} ; \mathbb{Z}_{2}\right)$, where $s$ runs over $\Sigma$. The sets $\Theta^{(0)}(\mathfrak{S})$ and $\Theta^{(2)}(\mathfrak{S})$ have canonical actions of $G \times\{ \pm 1\}$ and $G$, respectively. In addition, there is a canonical map $p_{\mathfrak{S}}: \Theta^{(0)}(\mathfrak{S}) \rightarrow \Theta^{(2)}(\mathfrak{S})$; for a generator $x$ of $H_{k}\left(X_{s}, X_{s} ; \mathbb{Z}\right), p_{\mathfrak{S}}(x)$ is the generator of $H_{k}\left(X_{s}, X_{s} ; \mathbb{Z}_{2}\right)$. We have a natural one-to-one correspondence from $\Sigma$ to $\Theta^{(2)}(\mathfrak{S})$. Thus we often identify $\Theta^{(2)}(\mathfrak{S})$ with $\Sigma$ as $G$-sets. On the other hand, we may not have a $(G \times\{ \pm 1\})$ bijection from $\Theta^{(0)}(\mathfrak{S})$ to $\Sigma_{+} \times\{ \pm 1\}$, although there is a non-equivariant bijection between these sets. Let $\rho_{\mathfrak{S}}$ denote the map $\Theta^{(2)}(\mathfrak{S})=\Sigma \rightarrow \mathcal{S}(G)$ defined by

Let $\boldsymbol{\Theta}(\mathfrak{S})$ denote the datum

$$
\rho_{\mathfrak{S}}(s)=\bigcap_{x \in X_{s}} G_{x} \quad(s \in \Sigma) .
$$

$$
\left(\Theta^{(0)}(\mathfrak{S}), \Theta^{(2)}(\mathfrak{S}), p_{\mathfrak{S}}, \rho_{\mathfrak{S}}\right) .
$$

Set

$$
\begin{gathered}
\widetilde{Q}=Q_{\widetilde{X}}\left(=\left\{g \in \widetilde{G}(2) \mid \operatorname{dim} \widetilde{X}^{g}=k-1\right\}\right), \\
\widetilde{S}=S_{\widetilde{X}}\left(=\left\{g \in \widetilde{G}(2) \mid \operatorname{dim} \widetilde{X}^{g}=k\right\}\right), \\
(\widetilde{Q})_{R}=R[\widetilde{Q}]+\{x-\lambda \bar{x} \mid x \in R[\widetilde{G}]\}, \quad(\widetilde{S})_{R}=R[\widetilde{S}]+\{x+\lambda \bar{x} \mid x \in R[\widetilde{G}]\} .
\end{gathered}
$$

Then

$$
\widetilde{\boldsymbol{A}}=\boldsymbol{A}_{\widetilde{X}}=\left(R[\widetilde{G}],(\bar{\cdot}, \lambda),(\widetilde{S})_{R}, \widetilde{G}, R[\widetilde{S}],(\widetilde{Q})_{R}+R[\widetilde{S}]\right)
$$

is a double parameter algebra.

Let $\mathfrak{S}=\left\{X_{s} \mid s \in \Sigma\right\}$ be a $k$-singular structure of $X$ as above. Consider the set

$$
\widetilde{\mathfrak{S}}=\left\{\widetilde{X}_{t} \mid t \in \widetilde{\Sigma}\right\}
$$

of all connected components $\widetilde{X}_{t}$ of $\pi_{\widetilde{X}, X}^{-1}\left(X_{s}\right), s \in \Sigma$, where $\pi_{\widetilde{X}, X}$ is the canonical projection $\widetilde{X} \rightarrow X$. Here we have canonical surjections $\widetilde{\mathfrak{S}} \rightarrow \mathfrak{S}$ and $\widetilde{\Sigma} \rightarrow \Sigma$. We call $\widetilde{\mathfrak{S}}$ the $k$-singular structure of $\widetilde{X}$ induced from $\mathfrak{S}$. Note that $\widetilde{X}$ and $\widetilde{X}_{t}$ are possibly non-compact. The index set $\widetilde{\Sigma}$ decomposes into the disjoint union of $\widetilde{\Sigma}_{+}$and $\widetilde{\Sigma}_{-}$consisting of all elements $t \in \widetilde{\Sigma}$ such that $\widetilde{X}_{t}$ is orientable and non-orientable, respectively. Let $\Theta^{(0)}(\widetilde{\mathfrak{S}})$ denote the set of all generators of 
$H_{k}^{\text {loc.fin. }}\left(\widetilde{X}_{t}, \partial \widetilde{X}_{t} ; \mathbb{Z}\right)$, where $t$ runs over $\widetilde{\Sigma}_{+}$, and let $\Theta^{(2)}(\widetilde{\mathfrak{S}})$ denote the set of all generators of $H_{k}^{\text {loc.fin. }}\left(\widetilde{X}_{t}, \partial \widetilde{X}_{t} ; \mathbb{Z}_{2}\right)$, where $t$ runs over $\widetilde{\Sigma}$. The sets $\Theta^{(0)}(\widetilde{\mathfrak{S}})$ and $\Theta^{(2)}(\widetilde{\mathfrak{S}})$ have canonical actions of $\widetilde{G} \times\{ \pm 1\}$ and $\widetilde{G}$, respectively. In addition, we have the canonical map $p_{\widetilde{\mathfrak{S}}}: \Theta^{(0)}(\widetilde{\mathfrak{S}}) \rightarrow \Theta^{(2)}(\widetilde{\mathfrak{S}})$. Define the map

$$
\rho_{\widetilde{\mathfrak{S}}}: \Theta^{(2)}(\widetilde{\mathfrak{S}})=\widetilde{\mathfrak{S}}=\widetilde{\Sigma} \rightarrow \mathcal{S}(\widetilde{G}) \quad \text { by } \quad \rho_{\widetilde{\mathfrak{S}}}(t)=\bigcap_{x \in \widetilde{X}_{t}} \widetilde{G}_{x} .
$$

Let $\boldsymbol{\Theta}(\widetilde{\mathfrak{S}})$ denote the datum

$$
\left(\Theta^{(0)}(\widetilde{\mathfrak{S}}), \Theta^{(2)}(\widetilde{\mathfrak{S}}), p_{\widetilde{\mathfrak{S}}}, \rho_{\widetilde{\mathfrak{S}}}\right) .
$$

The next lemma is well-known.

Lemma 3.1. Let $\boldsymbol{f}=(f, b)$ be a $G$-framed map and $\mathfrak{S}$ a $k$-singular structure of $X$ as above. Suppose the map $f:(X, \partial X) \rightarrow(Y, \partial Y)$ has degree one. Then $\boldsymbol{f}$ can be converted to a G-framed map $f^{\prime}=\left(f^{\prime}, b^{\prime}\right)$, where $f^{\prime}:\left(X^{\prime}, \partial X^{\prime}\right) \rightarrow(Y, \partial Y)$ and

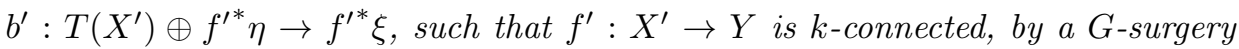
on $X$ relative to $X_{\operatorname{sing}} \cup X_{\mathfrak{S}} \cup \partial X$.

First, note that the degree of the resulting map $f^{\prime}:\left(X^{\prime}, \partial X^{\prime}\right) \rightarrow(Y, \partial Y)$ above is 1 . Second, note that if $f: X \rightarrow Y$ is $k$-connected then the mapping cylinder $M_{\widetilde{f}}$ of $\widetilde{f}: \widetilde{X} \rightarrow \widetilde{Y}$ is the universal covering space of the mapping cylinder $M_{f}$ of $f: X \rightarrow Y$, the group $\pi_{k+1}(\widetilde{f})$ can be identified with $\pi_{k+1}(f)$, and the canonical homomorphism $\pi_{k+1}(\widetilde{f}) \rightarrow K_{k}(\widetilde{f} ; \mathbb{Z})$ is an isomorphism, where $\pi_{k+1}(\widetilde{f})=\pi_{k+1}\left(M_{\widetilde{f}}, \widetilde{X}\right)$ and

$$
K_{k}(\widetilde{f} ; \mathbb{Z})=\operatorname{Ker}\left[\widetilde{f}_{*}: H_{k}(\widetilde{X} ; \mathbb{Z}) \rightarrow H_{k}(\widetilde{Y} ; \mathbb{Z})\right] .
$$

Now let $R$ be $\mathbb{Z}$ or $\mathbb{Z}_{(p)}$ for a prime $p$. We denote by $\mathcal{P}_{p}(G)$ the set of all subgroups of $G$ with $p$-power order. Thus we have

$$
\mathcal{P}(G)=\bigcup_{p \text { prime }} \mathcal{P}_{p}(G) .
$$

Let $\boldsymbol{f}=(f, b), f:(X, \partial X) \rightarrow(Y, \partial Y)$ be a $G$-framed map and $\mathfrak{S}$ a $k$-singular structure of $X$ as above. Then let $\widetilde{f}=(\widetilde{f}, \widetilde{b}), \widetilde{f}:(\widetilde{X}, \partial \widetilde{X}) \rightarrow(\widetilde{Y}, \partial \widetilde{Y})$, denote the $\widetilde{G}$-framed map induced from $\boldsymbol{f}$, where $\widetilde{X}$ and $\widetilde{Y}$ are the universal covering spaces of $X$ and $Y$, respectively. Let $\widetilde{\mathfrak{S}}$ denote the induced $k$-singular structure of $\widetilde{X}$.

Definition 3.2. Let $f$ be the $G$-framed map above. We define the $R[\widetilde{G}]$-module $M(\widetilde{f} ; R)$ by

$$
M(\widetilde{f} ; R)=\pi_{k+1}(\widetilde{f}) \otimes R .
$$

We call $\boldsymbol{f}$ a $(G, R)$-surgery map if the following conditions are fulfilled: 
(3.2.1) $f: X \rightarrow Y$ is of degree one.

(3.2.2) $f: X \rightarrow Y$ is 1-connected.

(3.2.3) $f_{*}: H_{j}(X ; R) \rightarrow H_{j}(Y ; R), j<k$, are all isomorphisms, and $f_{*}: H_{k}(X ; R)$ $\rightarrow H_{k}(Y ; R)$ is surjective.

(3.2.4) $\partial f_{*}: H_{j}(\partial X ; R) \rightarrow H_{j}(\partial Y ; R), j \leq n-1$, are all isomorphisms.

(3.2.5) $f: X \rightarrow Y$ is $k$-connected, or the canonical map $M(\widetilde{f} ; R) \otimes_{R[\widetilde{G}]} R[G] \rightarrow$ $K_{k}(f ; R)$ is an isomorphism, where

$$
K_{k}(f ; R)=\operatorname{Ker}\left[f_{*}: H_{k}(X ; R) \rightarrow H_{k}(Y ; R)\right] .
$$

(3.2.6) In the case $R=\mathbb{Z}, f^{P}: X^{P} \rightarrow Y^{P}$ are $\mathbb{Z}_{q}$-homology equivalences for all subgroups $P \in \mathcal{P}(G)$ with $P \neq\{e\}$, and primes $q$ dividing $|P|$. In the case $R=\mathbb{Z}_{(p)}, f^{P}: X^{P} \rightarrow Y^{P}$ are $\mathbb{Z}_{p}$-homology equivalences for all $P \in \mathcal{P}_{p}(G)$ with $P \neq\{e\}$.

(3.2.7) $\chi\left(X^{g}\right)=\chi\left(Y^{g}\right)$ for all $g \in G, g \neq e$.

We have the Poincaré pairing

$$
H_{k}^{\text {loc.fin. }}(\tilde{X}, \partial \widetilde{X} ; \mathbb{Z}) \times H_{k}(\widetilde{X} ; \mathbb{Z}) \rightarrow \mathbb{Z} .
$$

Passing along the canonical homomorphisms

$$
\pi_{k+1}(\tilde{f}) \rightarrow H_{k+1}\left(M_{\tilde{f}}, \tilde{X} ; \mathbb{Z}\right) \rightarrow K_{k}(\tilde{f} ; \mathbb{Z}) \subset H_{k}(\tilde{X} ; \mathbb{Z}) \rightarrow H_{k}^{\text {loc.fin. }}(\tilde{X}, \partial \tilde{X} ; \mathbb{Z})
$$

we obtain the intersection form $\widetilde{B}_{0}: M(\widetilde{f} ; R) \times M(\widetilde{f} ; R) \rightarrow R$, and hence the $\widetilde{G}$-equivariant intersection form

$$
\widetilde{B}: M(\widetilde{f} ; R) \times M(\widetilde{f} ; R) \rightarrow R[\widetilde{G}] ; \widetilde{B}(x, y)=\sum_{g \in \widetilde{G}} \widetilde{B}_{0}\left(x, g^{-1} y\right) g
$$

Let $x \in \pi_{k+1}(\tilde{f})$. Then $x$ is represented by a commutative diagram

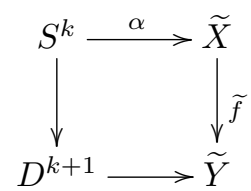

By virtue of this diagram and the bundle isomorphism $b$, the induced bundle $\alpha^{*} T(\widetilde{X})$ is stably trivial. Thus $x$ is represented by an immersion $\alpha: S^{k} \rightarrow \widetilde{X}$ with trivial normal bundle. Let $g$ be an element in $\widetilde{G}$ of order 2 satisfying $\operatorname{dim} \widetilde{X}^{g} \leq k-2$. Then the regular homotopy classes of immersions $S^{k} \rightarrow \widetilde{X}$ correspond in a oneto-one way to the regular homotopy classes of immersions $S^{k} \rightarrow \widetilde{X} \backslash \widetilde{X}^{g}$. Hence 
Theorem 5.2 of [18] provides the $\langle g\rangle$-equivariant self-intersection form

$$
\widetilde{q}_{\langle g\rangle}: \pi_{k+1}(\widetilde{f}) \rightarrow \mathbb{Z}[\langle g\rangle] /\{a-\lambda \bar{a} \mid a \in \mathbb{Z}[\langle g\rangle]\} .
$$

Assembling the data of the $\widetilde{G}$-equivariant intersection form $\widetilde{B}$ and the $\langle g\rangle$-equivariant self-intersection forms $\widetilde{q}_{\langle g\rangle}$ (cf. [2, Definition 4.11]), we obtain the $\widetilde{G}$-equivariant self-intersection form

$$
\widetilde{q}: M(\widetilde{f} ; R) \rightarrow R[\widetilde{G}] /\left(\left(Q_{\widetilde{X}}\right)_{R}+R\left[S_{\widetilde{X}}\right]\right) \quad(\text { cf. }[2, \text { p. } 567, \ell .3]) .
$$

For a generator $\alpha \in H_{k}^{\text {loc.fin. }}\left(\widetilde{X}_{t}, \partial \widetilde{X}_{t} ; \mathbb{Z}\right)$, where $t \in \widetilde{\Sigma}_{+}$, we have the element

$$
j_{*} \alpha \in H_{k}^{\text {loc.fin. }}(\widetilde{X}, \partial \widetilde{X} ; \mathbb{Z}),
$$

where $j_{*}: H_{k}^{\text {loc.fin. }}\left(\widetilde{X}_{t}, \partial \widetilde{X}_{t} ; \mathbb{Z}\right) \rightarrow H_{k}^{\text {loc.fin. }}(\tilde{X}, \partial \widetilde{X} ; \mathbb{Z})$ is the canonical homomorphism. Via the intersection paring (or the Poincaré pairing up to sign)

$$
H_{k}^{\text {loc.fin. }}(\tilde{X}, \partial \widetilde{X} ; \mathbb{Z}) \times H_{k}(\widetilde{X} ; \mathbb{Z}) \rightarrow \mathbb{Z}
$$

and the canonical map $M(\tilde{f} ; \mathbb{Z}) \rightarrow H_{k}(\tilde{X} ; \mathbb{Z}), j_{*} \alpha$ determines an element

$$
\widetilde{\theta}^{(0)}(\alpha) \in M(\tilde{f} ; \mathbb{Z})^{\#}, \quad \text { where } \quad M(\widetilde{f} ; \mathbb{Z})^{\#}=\operatorname{Hom}_{\mathbb{Z}[\widetilde{G}]}(M(\tilde{f} ; \mathbb{Z}), \mathbb{Z}[\widetilde{G}]) .
$$

Thus we obtain the $(\widetilde{G} \times\{ \pm 1\})$-map

$$
\widetilde{\theta}^{(0)}: \Theta^{(0)}(\widetilde{\mathfrak{S}}) \rightarrow M(\widetilde{f} ; R)^{\#}, \quad \text { where } \quad M(\widetilde{f} ; R)^{\#}=\operatorname{Hom}_{R[\widetilde{G}]}(M(\widetilde{f} ; R), R[\widetilde{G}]) .
$$

Similarly we obtain the $\widetilde{G}$-map

$$
\widetilde{\theta}^{(2)}: \Theta^{(2)}(\widetilde{\mathfrak{S}}) \rightarrow M(\widetilde{f} ; R / 2 R)^{\#},
$$

where

$$
M(\tilde{f} ; R / 2 R)^{\#}=\operatorname{Hom}_{R / 2 R[\widetilde{G}]}(M(\tilde{f} ; R / 2 R), R / 2 R[\widetilde{G}]) .
$$

Putting all this together, we obtain the surgery module

$$
M_{\widetilde{\boldsymbol{f}}, \widetilde{\mathfrak{S}}}=\left(M(\widetilde{f} ; R), \widetilde{B}, \widetilde{q}, \widetilde{\theta}^{(0)}, \widetilde{\theta}^{(2)}\right) .
$$
diagram

By the hypothesis $M(\tilde{f} ; R) \otimes_{R[\widetilde{G}]} R[G]=K_{k}(f ; R)$, we obtain the commutative

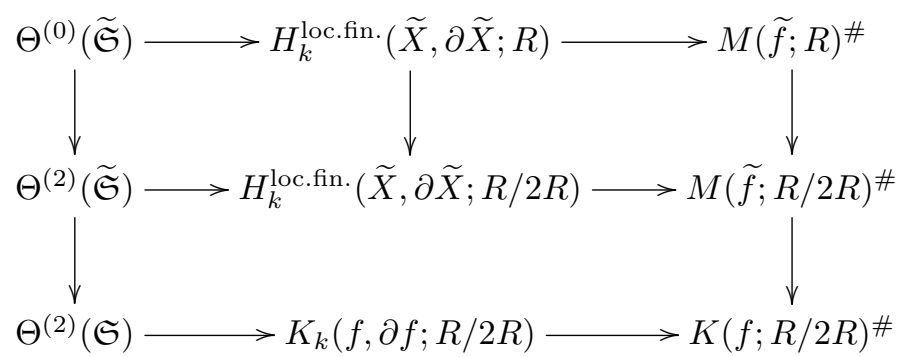


Moreover we note

$$
\begin{aligned}
& K_{k}(f, \partial f ; R)=K_{k}(f ; R), \quad K_{k}(f, \partial f ; R / 2 R)=K_{k}(f ; R / 2 R), \\
& K_{k}(f ; R)^{\#}=K_{k}(f ; R) \quad \text { and } \quad K_{k}(f ; R / 2 R)^{\#}=K_{k}(f ; R / 2 R) .
\end{aligned}
$$

Thus by the hypothesis $M(\tilde{f} ; R) \otimes_{R[\widetilde{G}]} R[G]=K_{k}(f ; R)$, we obtain the surgery module

$$
M_{\boldsymbol{f}, \mathfrak{S}}=M_{\widetilde{\boldsymbol{f}, \widetilde{S}}} \otimes_{R[\widetilde{G}]} R[G]=\left(K_{k}(f ; R), B_{f}, q_{\boldsymbol{f}}, \theta^{(0)}, \theta^{(2)}\right),
$$

where

$$
B_{f}: K_{k}(f ; R) \times K_{k}(f ; R) \rightarrow R[G]
$$

is the $G$-equivariant intersection form,

$$
q_{\boldsymbol{f}}: K_{k}(f ; R) \rightarrow R[G] /\left((Q)_{R}+R[S]\right)
$$

is the $G$-equivariant self-intersection form, and

$$
\begin{aligned}
& \theta^{(0)}: \Theta^{(0)}(\mathfrak{S}) \rightarrow K_{k}(f ; R)^{\#}=K_{k}(f ; R), \\
& \theta^{(2)}: \Theta^{(2)}(\mathfrak{S}) \rightarrow K_{k}(f ; R / 2 R)^{\#}=K_{k}(f ; R / 2 R)
\end{aligned}
$$

are positioning maps (cf. [2, §5, pp. 563-564]).

By similar arguments to $[2$, p. $575, \ell .24-$ p. $578, \ell .2]$, we obtain the next lemma.

Lemma 3.3. Let $f$ be a $(G, R)$-surgery map and $\mathfrak{S}$ a $k$-singular structure as above. If there exists an $R[\widetilde{G}]$-submodule $\widetilde{L}$ of $M(\widetilde{f} ; R)$ satisfying the conditions below then $f$ can be converted to a $(G, R)$-surgery map $f^{\prime}=\left(f^{\prime}, b^{\prime}\right)$, where $f^{\prime}$ : $\left(X^{\prime}, \partial X^{\prime}\right) \rightarrow(Y, \partial Y)$ and $b^{\prime}: T\left(X^{\prime}\right) \oplus f^{\prime *} \eta \rightarrow f^{\prime *} \xi$, such that $f^{\prime}: X^{\prime} \rightarrow Y$ is an $R$-homology equivalence via $G$-surgery on $X$ relative to $X_{\text {sing }} \cup X_{\mathfrak{S}} \cup \partial X$.

$(3.3 .1) \widetilde{\theta}^{(0)}(\alpha)(\widetilde{L})=0$ for all $\alpha \in \Theta^{(0)}(\widetilde{\mathfrak{S}})$ and $\widetilde{\theta}^{(2)}(\beta)(\widetilde{L})=0$ for all $\beta \in \Theta^{(2)}(\widetilde{\mathfrak{S}})$.

(3.3.2) $\widetilde{B}(\widetilde{L}, \widetilde{L})=0$.

(3.3.3) $\widetilde{q}(\widetilde{L})=0$.

(3.3.4) The canonical image $L$ in $K_{k}(f ; R)$ of $\widetilde{L}$ is an $R[G]$-free direct summand of $K_{k}(f ; R)$ of half the rank, i.e. $2 \cdot \operatorname{rank}_{R} L=\operatorname{rank}_{R} K_{k}(f ; R)$.

Lemma 3.4. Let $p$ be a prime and $R=\mathbb{Z}_{(p)}$. Let $\boldsymbol{f}=(f, b)$ be $a(G, R)$-surgery map and $\mathfrak{S}$ a $k$-singular structure of $X$ as above. Suppose the following.

(3.4.1) $\pi_{1}(X)$ is finite and $\left|\pi_{1}(X)\right|$ is prime to $p$.

(3.4.2) the canonical homomorphism $\widetilde{G} \rightarrow G$ has a splitting, i.e. $\widetilde{G}=\pi_{1}(X) \rtimes G$.

(3.4.3) $f: X \rightarrow Y$ is k-connected.

(3.4.4) $\pi_{\widetilde{X}, X}\left(\widetilde{X}_{t}\right)$ are orientable for all $t \in \widetilde{\Sigma}_{+}$. 
If the module

$$
\boldsymbol{M}_{\boldsymbol{f}, \mathfrak{S}}=\left(K_{k}(f ; R), B_{f}, q_{\boldsymbol{f}}, \theta^{(0)}, \theta^{(2)}\right)
$$

has an $R[G]$-free Lagrangian $L$, then there exists a submodule $\widetilde{L}$ of $M(f ; R)$ satisfying the conditions (3.3.1)-(3.3.4).

Before proving this lemma, we give an important application of the two lemmas above. Let

$$
W_{n}\left(\boldsymbol{A}_{X}, \boldsymbol{\Theta}(\mathfrak{S})\right)_{\text {free }}
$$

denote the surgery obstruction group

$$
W_{n}\left(R, G, Q_{X}, S_{X}, \boldsymbol{\Theta}(\mathfrak{S})\right)_{\text {free }}
$$

defined in [2, p. 545, Definition 3.33]. In the case $R=\mathbb{Z}_{(p)}$, a $(G, R)$-surgery map $\boldsymbol{f}$ with $k$-singular structure $\mathfrak{S}$ determines the module $\boldsymbol{M}_{\boldsymbol{f}, \mathfrak{S}}$ above, and further the element $\sigma(\boldsymbol{f}, \mathfrak{S})$ of $W_{n}\left(\boldsymbol{A}_{X}, \boldsymbol{\Theta}(\mathfrak{S})\right)_{\text {free }}$ as the equivalence class of $\boldsymbol{M}_{\boldsymbol{f}, \mathfrak{S}}$. By Lemmas 3.3, 3.4 and [18, Lemma 5.5], we obtain the next theorem.

Theorem 3.5. Let $R=\mathbb{Z}_{(p)}$ for a prime $p, f a(G, R)$-surgery map and $\mathfrak{S}$ a $k$-singular structure satisfying the conditions (3.4.1)-(3.4.4). If $\sigma(\boldsymbol{f}, \mathfrak{S})=0$ in $W_{n}\left(\boldsymbol{A}_{X}, \boldsymbol{\Theta}(\mathfrak{S})\right)_{\text {free }}$ then $\boldsymbol{f}$ can be converted to $\boldsymbol{f}^{\prime}=\left(f^{\prime}, b^{\prime}\right)$ such that $f^{\prime}: X^{\prime} \rightarrow Y$ is an $R$-homology equivalence via a $G$-surgery on $X$ relative to $X_{\text {sing }} \cup X_{\mathfrak{S}} \cup \partial X$.

Proof of Lemma 3.4. Let $L$ be an $R[G]$-free Lagrangian of $\boldsymbol{M}_{\boldsymbol{f}, \mathfrak{S}}$. Let $\left\{x_{1}, \ldots, x_{m}\right\}$ be an $R[G]$-basis of $L$ and $\left\{y_{1}, \ldots, y_{m}\right\}$ be elements of $K_{k}(f ; R)$ such that

$$
B_{f}\left(x_{i}, y_{j}\right)=\delta_{i j}
$$

for $1 \leq i, j \leq m$. Thus $\left\{x_{1}, \ldots, x_{m}, y_{1}, \ldots, y_{m}\right\}$ is an $R[G]$-basis of $K_{k}(f ; R)$. Arbitrarily choose liftings $\widetilde{x}_{1}, \ldots, \widetilde{x}_{m}, \widetilde{y}_{1}, \ldots, \widetilde{y}_{m} \in M(\widetilde{f} ; R)$ of $x_{1}, \ldots, x_{m}, y_{1}, \ldots, y_{m}$, respectively. Define a map $\tau: K_{k}(f ; R) \rightarrow M(\widetilde{f} ; R)$ by

$$
\tau\left(\sum_{i}\left(a_{i} x_{i}+b_{i} y_{i}\right)\right)=\frac{1}{\left|\pi_{1}(X)\right|} \sum_{i} \sum_{h \in \pi_{1}(X)}\left(h a_{i} \widetilde{x_{i}}+h b_{i} \widetilde{y_{i}}\right) .
$$

This map is an $R[G]$-splitting of the canonical map $M(\tilde{f} ; R) \rightarrow K_{k}(f ; R)$. Clearly, $\pi_{1}(X)$ acts trivially on the image of $\tau$. Set

$$
\widetilde{L}=\tau(L) .
$$

That $\widetilde{B}(\widetilde{L}, \widetilde{L})=0$ and $\widetilde{q}(\widetilde{L})=0$ follows from Steps 1 and 2 in the proof of $[11$, Theorem 2.6].

Thus it suffices to show that $\widetilde{\theta}^{(0)}(\alpha)(\widetilde{L})=0$ for $\alpha \in \Theta^{(0)}(\widetilde{\mathfrak{S}})$, and $\widetilde{\theta}^{(2)}(\beta)(\widetilde{L})=0$ for $\beta \in \Theta^{(2)}(\widetilde{\mathfrak{S}})$. Let $\widetilde{\varepsilon}: R[\widetilde{G}] \rightarrow R$ and $\varepsilon: R[G] \rightarrow R$ be the 
homomorphisms of taking the coefficients of the identity elements of $\widetilde{G}$ and $G$, respectively. For $\alpha \in \Theta^{(0)}(\widetilde{\mathfrak{S}})$, let $[\alpha]$ denote the canonical image of $\alpha$ in $\Theta^{(0)}(\mathfrak{S})$ and let $\pi_{1}(X)_{\alpha}$ denote the isotropy subgroup of the $\pi_{1}(X)$-action on $\Theta^{(0)}(\widetilde{\mathfrak{S}})$ at the point $\alpha$. Then the canonical map $M(\tilde{f} ; R) \rightarrow K_{k}(f ; R)$ assigns $m \theta^{(0)}([\alpha])$ to $\widetilde{\theta}^{(0)}(\alpha)$ with $m=\left|\pi_{1}(X)_{\alpha}\right|$. Thus for $x \in L$, we get

$$
\begin{aligned}
\varepsilon\left(\theta^{(0)}([\alpha])(x)\right) & =\sum_{h \in \pi_{1}(X)} \widetilde{\varepsilon}\left(\frac{1}{m} \widetilde{\theta}^{(0)}(\alpha)\left(h^{-1} \tau(x)\right)\right) \\
& =\sum_{h \in \pi_{1}(X)} \widetilde{\varepsilon}\left(\frac{1}{m} \widetilde{\theta}^{(0)}(\alpha)(\tau(x))\right) \\
& =\left|\pi_{1}(X): \pi_{1}(X)_{\alpha}\right| \widetilde{\varepsilon}\left(\widetilde{\theta}^{(0)}(\alpha)(\tau(x))\right),
\end{aligned}
$$

and hence

$$
\widetilde{\varepsilon}\left(\widetilde{\theta}^{(0)}(\alpha)(\tau(x))\right)=\frac{\left|\pi_{1}(X)_{\alpha}\right|}{\left|\pi_{1}(X)\right|} \varepsilon\left(\theta^{(0)}([\alpha])(x)\right)=0
$$

Since

$$
\widetilde{\theta}^{(0)}(\alpha)(\tau(x))=\sum_{g \in \widetilde{G}} \widetilde{\varepsilon}\left(\widetilde{\theta}^{(0)}(\alpha)\left(\tau\left(g^{-1} x\right)\right)\right) g,
$$

the triviality $\theta^{(0)}([\alpha])(L)=0$ implies $\widetilde{\theta}^{(0)}(\alpha)(\tau(x))=0$.

We can similarly show that $\widetilde{\theta}^{(2)}(\beta)(\tau(x))=0$.

\section{§4. The Mackey structure of surgery obstruction groups}

In this section, let $R$ be a principal ideal domain, hence necessarily a commutative ring, with 1 satisfying the square condition, i.e.

$$
r \equiv r^{2} \bmod 2 R \quad \text { for each } r \in R .
$$

Let $\Theta$ be a finite $G$-set, $\rho: \Theta \rightarrow \mathcal{S}(G)$ a $G$-map, and $S$ a conjugation invariant subset of $G(2)$. The map $\mathcal{S}(G) \rightarrow \mathfrak{P}(S) ; H \mapsto S_{H}=S \cap H$, preserves intersection. Let $\mathrm{SGW}_{0}(R, G, S, \Theta)$ denote the special Grothendieck-Witt group defined in [10, p. 2358].

Lemma 4.1 ([10, Proposition 5.4]). If $\rho$ is $S$-injective then $\operatorname{SGW}_{0}(R, G, S, \Theta)$ is a commutative ring possibly without 1 , and moreover the canonical map

$$
\mathrm{SGW}_{0}(\mathbb{Z}, G, S, \Theta) \rightarrow \mathrm{SGW}_{0}(R, G, S, \Theta)
$$

of ring change is a ring homomorphism. If $\rho$ is $S$-bijective then $\mathrm{SGW}_{0}(R, G, S, \Theta)$ possesses the unit 1 . 
Let

$$
f: \mathcal{S}(G) \rightarrow \mathfrak{P}(\Theta) ; H \mapsto \Theta_{H},
$$

be an intersection preserving $\rho$-compatible $G$-map and let $w: G \rightarrow\{ \pm 1\}$ be a homomorphism. We denote by $w_{H}$ the restriction $\left.w\right|_{H}: H \rightarrow\{ \pm 1\}$.

Definition 4.2 (cf. [10, p. 2357]). For a $\Theta$-positioning Hermitian form $\boldsymbol{M}=$ $(M, B, \theta)$, where $M$ is an $R$-free $R[G]$-module, $B: M \times M \rightarrow R$ is a $G$-invariant (or $w$-invariant) symmetric bilinear form, and $\theta: \Theta \rightarrow M$ is a $G$-map, and for $s \in S, x \in M$, we define the trace $\Delta_{\theta}(s) \in M$ of $(\theta, \rho)$ at $s$ and the $\nabla$-invariant $\nabla_{\boldsymbol{M}}(x)(s) \in R / 2 R$ of $\boldsymbol{M}$ at $(x, s)$ by

$$
\Delta_{\theta}(s)=\sum_{t \in \Theta}\{\theta(t) \mid \rho(t) \ni s\}, \quad \nabla_{\boldsymbol{M}}(x)(s)=\left[B\left(\Delta_{\theta}(s)-x, s x\right)\right] .
$$

We remark that what we precisely need for the definition is $B: M \times M \rightarrow R / 2 R$ rather than $B: M \times M \rightarrow R$.

Lemma 4.3. Let $H$ and $K$ be subgroups of $G$ and let $\varphi=(\varphi, \psi)$ be a pair consisting of a monomorphism $\varphi: H \rightarrow K$ which is a composition of inclusion and conjugation and the associated injective $\varphi$-map $\psi: \Theta_{H} \rightarrow \Theta_{K}$. Let $g_{1}, \ldots, g_{m} \in K$ be a complete set of representatives of $K / \varphi(H)$. Further let $\boldsymbol{M}=(M, B, \alpha)$ be a positioning Hermitian module, where $M$ is an $R$-free $R[H]$-module, $B: M \times M \rightarrow R$ is an $H$-invariant (or $w_{H}$-invariant) symmetric bilinear form, and $\alpha: \Theta_{H} \rightarrow M$ is an $H$-map. Then the $\nabla$-invariant of the induced module $\boldsymbol{M}^{\prime}=\boldsymbol{\varphi}_{\#} M$ satisfies

$$
\nabla_{\boldsymbol{M}^{\prime}}\left(g_{i} \otimes_{\varphi} x\right)\left(s^{\prime}\right)= \begin{cases}\nabla_{\boldsymbol{M}}(x)\left(\varphi^{-1}\left(g_{i}^{-1} s^{\prime} g_{i}\right)\right) & \left(g_{i}^{-1} s^{\prime} g_{i} \in \varphi(H)\right), \\ 0 & \left(g_{i}^{-1} s^{\prime} g_{i} \notin \varphi(H)\right),\end{cases}
$$

for $x \in M$ and $s^{\prime} \in S_{K}=S \cap K$.

Proof. By definition, $\boldsymbol{M}^{\prime}=\left(M^{\prime}, B^{\prime}, \alpha^{\prime}\right)$ is given by $M^{\prime}=R[K] \otimes_{R[H], \varphi} M$,

$$
\begin{aligned}
& B^{\prime}\left(g_{j} \otimes_{\varphi} x, g_{k} \otimes_{\varphi} y\right)=\delta_{j k} B(x, y), \quad \text { and } \\
& \alpha^{\prime}\left(t^{\prime}\right)=\sum_{(i, t)}\left\{g_{i} \otimes \alpha(t) \mid t \in \Theta_{H}, g_{i} \psi(t)=t^{\prime}\right\}
\end{aligned}
$$

where $x, y \in M, t^{\prime} \in \Theta_{K}$. Let $s^{\prime} \in S_{K}$. We have

$$
\nabla_{\boldsymbol{M}^{\prime}}\left(g_{i} \otimes_{\varphi} x\right)\left(s^{\prime}\right)=B^{\prime}\left(\Delta_{\alpha^{\prime}}\left(s^{\prime}\right)-g_{i} \otimes_{\varphi} x, s^{\prime}\left(g_{i} \otimes_{\varphi} x\right)\right) .
$$


Moreover the following equalities hold:

$$
\begin{aligned}
B^{\prime} & \left(\Delta_{\alpha^{\prime}}\left(s^{\prime}\right), s^{\prime}\left(g_{i} \otimes_{\varphi} x\right)\right)=B^{\prime}\left(\Delta_{\alpha^{\prime}}\left(s^{\prime}\right), g_{i} \otimes_{\varphi} x\right) \\
& =\sum_{t^{\prime} \in \Theta_{K}}\left\{B^{\prime}\left(\psi_{\#} \alpha\left(t^{\prime}\right), g_{i} \otimes_{\varphi} x\right) \mid \rho_{K}\left(t^{\prime}\right) \ni s^{\prime}\right\} \\
& =\sum_{t^{\prime} \in \Theta_{K}} \sum_{j, t}\left\{B^{\prime}\left(g_{j} \otimes_{\varphi} \alpha(t), g_{i} \otimes_{\varphi} x\right) \mid t \in \Theta_{H}, g_{j} \psi(t)=t^{\prime}, g_{j} \varphi\left(\rho_{H}(t)\right) g_{j}^{-1} \ni s^{\prime}\right\} \\
& =\sum_{t^{\prime} \in \Theta_{K}} \sum_{t}\left\{B(\alpha(t), x) \mid t \in \Theta_{H}, g_{i} \psi(t)=t^{\prime}, g_{i} \varphi\left(\rho_{H}(t)\right) g_{i}^{-1} \ni s^{\prime}\right\} \\
& =\sum_{t \in \Theta_{H}}\left\{B(\alpha(t), x) \mid \varphi\left(\rho_{H}(t)\right) \ni g_{i}^{-1} s^{\prime} g_{i}\right\} \\
& =\sum_{t \in \Theta_{H}}\left\{B(\alpha(t), x) \mid \rho_{H}(t) \ni \varphi^{-1}\left(g_{i}^{-1} s^{\prime} g_{i}\right)\right\} .
\end{aligned}
$$

On the other hand, we have

$$
B^{\prime}\left(g_{i} \otimes_{\varphi} x, s^{\prime}\left(g_{i} \otimes_{\varphi} x\right)\right)= \begin{cases}B\left(x, \varphi^{-1}\left(g_{i}^{-1} s^{\prime} g_{i}\right) x\right) & \left(g_{i}^{-1} s^{\prime} g_{i} \in \varphi(H)\right), \\ 0 & \left(g_{i}^{-1} s^{\prime} g_{i} \notin \varphi(H)\right) .\end{cases}
$$

Thus we obtain

$$
\nabla_{\boldsymbol{M}^{\prime}}\left(g_{i} \otimes_{\varphi} x\right)\left(s^{\prime}\right)= \begin{cases}\nabla_{\boldsymbol{M}}(x)\left(\varphi^{-1}\left(g_{i}^{-1} s^{\prime} g_{i}\right)\right) & \left(g_{i}^{-1} s^{\prime} g_{i} \in \varphi(H)\right), \\ 0 & \left(g_{i}^{-1} s^{\prime} g_{i} \notin \varphi(H)\right) .\end{cases}
$$

Lemma 4.4. If $f: \mathcal{S}(G) \rightarrow \mathfrak{P}(\Theta)$ is $(\rho, S)$-saturated then the correspondence

$$
H \mapsto \operatorname{SGW}_{0}\left(R, H, S_{H}, \Theta_{H}\right) \quad(H \in \mathcal{S}(G))
$$

affords a Mackey functor.

Proof. This follows from the proof of [10, Proposition 11.2] with a modification using Lemma 4.3.

Lemma 4.5 ([10, Theorem 11.3]). If $\rho: \Theta \rightarrow \mathcal{S}(G)$ is $S$-bijective and $f$ is $(\rho, S)$ saturated then the correspondence

$$
H \mapsto \operatorname{SGW}_{0}\left(R, H, S_{H}, \Theta_{H}\right) \quad(H \in \mathcal{S}(G))
$$

affords a Green functor. Moreover, the canonical homomorphisms

$$
\mathrm{SGW}_{0}\left(\mathbb{Z}, H, S_{H}, \Theta_{H}\right) \rightarrow \mathrm{SGW}_{0}\left(R, H, S_{H}, \Theta_{H}\right)
$$

of ring change afford a natural transformation of Green functors. 
Let $w: G \rightarrow\{ \pm 1\}$ be a homomorphism and let $\div$ denote the anti-involution on $\mathbb{Z}[G]$ associated with $w$. Let $\lambda=(-1)^{k}$. Then $\left(^{-}, \lambda\right)$ is an anti-structure of $\mathbb{Z}[G]$. Let $Q$ be a conjugation invariant subset of $G(2)$. Suppose

$$
S \subset G(2)^{\lambda}=\{g \in G(2) \mid g=\lambda \bar{g}\}, \quad Q \subset G(2)^{-\lambda}=\{g \in G(2) \mid g=-\lambda \bar{g}\} .
$$

Then we obtain the double parameter algebra

$$
\boldsymbol{A}=\left(R[G],\left({ }^{-}, \lambda\right),(S)_{R}, G, R[S],(Q)_{R}+R[S]\right)
$$

in the sense of $\left[2\right.$, Definition 2.5]. Let $\Theta^{(0)}$ and $\Theta^{(2)}$ be a finite $(G \times\{ \pm 1\})$ set and a finite $G$-set, respectively and let $p_{\Theta^{(0)}}: \Theta^{(0)} \rightarrow \Theta^{(2)}$ be a $G$-map. Throughout this paper we assume that $\{ \pm 1\}$ acts freely on $\Theta^{(0)}$ and $p_{\Theta^{(0)}}^{-1}\left(p_{\Theta^{(0)}}(t)\right)$ coincides with $\{t,-t\}$ for all $t \in \Theta^{(0)}$. Let $\rho_{\Theta^{(2)}}: \Theta^{(2)} \rightarrow \mathcal{S}(G)$ be a $G$-map and set $\boldsymbol{\Theta}=\left(\Theta^{(0)}, \Theta^{(2)}, p_{\Theta^{(0)}}, \rho_{\Theta^{(2)}}\right)$. We use the notation

$$
W_{n}(\boldsymbol{A}, \boldsymbol{\Theta})_{\text {free }}=W_{n}(R, G, Q, S, \boldsymbol{\Theta})_{\text {free }}, \quad W_{n}(\boldsymbol{A}, \boldsymbol{\Theta})_{\text {proj }}=W_{n}(R, G, Q, S, \boldsymbol{\Theta})_{\text {proj }},
$$

where $n=2 k$, defined in [2, Definition 3.33]

Let $\Theta$ be a finite $G$-set and $\rho: \Theta \rightarrow \mathcal{S}(G)$ a $G$-map. Let $\gamma: \Theta^{(2)} \rightarrow \Theta$ be a $G$-map such that the diagram

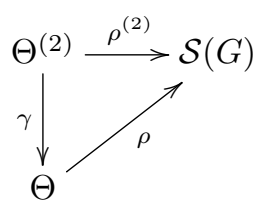

commutes and

$$
\gamma\left(\Theta^{(2)}\right)=\Theta
$$

Lemma 4.6. If $\rho: \Theta \rightarrow \mathcal{S}(G)$ is $S$-bijective then $W_{n}(\boldsymbol{A}, \boldsymbol{\Theta})_{\text {free }}$ is a module over $\mathrm{SGW}_{0}(R, G, S, \Theta)$.

Proof. Let $\boldsymbol{M}_{1}=\left(M_{1}, B_{1}, \alpha_{1}\right)$ be a $\Theta$-positioning, non-singular Hermitian $R[G]$ module with trivial $\nabla$-invariant, where $M$ is an $R$-free $R[G]$-module, $B_{1}: M_{1} \times M_{1}$ $\rightarrow R$ and $\alpha_{1}: \Theta \rightarrow M$. Let $\boldsymbol{M}_{2}=\left(M_{2}, B_{2}, q_{2}, \alpha^{(0)}, \alpha^{(2)}\right)$ be an object in $\nabla \mathcal{Q}(\boldsymbol{A}, \boldsymbol{\Theta})$ defined in [2, p. 535] such that $M_{2}$ is a stably $R[G]$-free module, where $B_{2}$ : $M_{2} \times M_{2} \rightarrow R[G], q_{2}: M_{2} \rightarrow R[G] /\left((Q)_{R}+R[S]\right), \alpha^{(0)}: \Theta^{(0)} \rightarrow M_{2}$, and $\alpha^{(2)}: \Theta^{(2)} \rightarrow M_{2} / 2 M_{2}$. Then we define

$$
\boldsymbol{M}=\boldsymbol{M}_{1} \cdot \boldsymbol{M}_{2}=\left(M, B, q, \theta^{(0)}, \theta^{(2)}\right) \in \mathcal{Q}(\boldsymbol{A}, \boldsymbol{\Theta})
$$


as follows. The triple $(M, B, q)$ is described in [10, §9]. The map $\theta^{(0)}: \Theta^{(0)} \rightarrow$ $M=M_{1} \otimes_{R} M_{2}$ is given by

$$
\theta^{(0)}(t)=\alpha_{1}\left(\gamma\left(p_{\Theta^{(0)}}(t)\right)\right) \otimes_{R} \alpha^{(0)}(t) \quad \text { for } t \in \Theta^{(0)},
$$

and the map $\theta^{(2)}: \Theta^{(2)} \rightarrow M / 2 M$ is given by

$$
\theta^{(2)}(t)=\alpha_{1}(\gamma(t)) \otimes_{R} \alpha^{(2)}(t)
$$

for $t \in \Theta^{(2)}$. It is easy to verify the $\nabla$-triviality of $\boldsymbol{M}$, i.e. $\boldsymbol{M} \in \nabla \mathcal{Q}(\boldsymbol{A}, \boldsymbol{\Theta})$. The correspondence $\left(\boldsymbol{M}_{1}, \boldsymbol{M}_{2}\right) \mapsto \boldsymbol{M}$ affords the module structure

$$
\operatorname{SGW}_{0}(R, G, S, \Theta) \times W_{n}(\boldsymbol{A}, \boldsymbol{\Theta})_{\text {free }} \mapsto W_{n}(\boldsymbol{A}, \boldsymbol{\Theta})_{\text {free }}
$$

In this section we set

$$
Q_{H}=Q \cap H \quad \text { for } H \in \mathcal{S}(G) .
$$

Then the map $\mathcal{S}(G) \rightarrow \mathfrak{P}(Q) ; H \mapsto Q_{H}$, preserves intersection.

We regard $\mathcal{S}(G)$ as a $(G \times\{ \pm 1\})$-set, with the trivial $\{ \pm 1\}$-action. Let $\boldsymbol{f}_{\boldsymbol{\Theta}}=$ $\left(f_{\Theta^{(0)}}, f_{\Theta^{(2)}}\right)$ be a pair of an intersection preserving $(G \times\{ \pm 1\})$-map $f_{\Theta^{(0)}}: \mathcal{S}(G) \rightarrow$ $\mathfrak{P}\left(\Theta^{(0)}\right) ; H \mapsto \Theta_{H}^{(0)}$, and an intersection preserving $\rho_{\Theta^{(2)}}$-compatible $G$-map $f_{\Theta^{(2)}}$ : $\mathcal{S}(G) \rightarrow \mathfrak{P}\left(\Theta^{(2)}\right) ; H \mapsto \Theta_{H}^{(2)}$, satisfying

$$
p_{\Theta(0)}\left(\Theta_{H}^{(0)}\right) \subset \Theta_{H}^{(2)}
$$

for $H \in \mathcal{S}(G)$. Define $p_{\Theta_{H}^{(0)}}: \Theta_{H}^{(0)} \rightarrow \Theta_{H}^{(2)}$ as the restriction of $p_{\Theta^{(0)}}$, and $\rho_{\Theta_{H}^{(2)}}$ : $\Theta_{H}^{(2)} \rightarrow \mathcal{S}(H)$ as the restriction of $\rho_{\Theta(2)}$. Then we obtain the double parameter algebras

$$
\boldsymbol{A}_{H}=\left(R[H],(\bar{\cdot}, \lambda),\left(S_{H}\right)_{R}, H, R\left[S_{H}\right],\left(Q_{H}\right)_{R}+R\left[S_{H}\right]\right),
$$

where $S_{H}=S \cap H$, and the positioning data

$$
\Theta_{H}=\left(\Theta_{H}^{(0)}, \Theta_{H}^{(2)}, p_{\Theta_{H}^{(0)}}, \rho_{\Theta_{H}^{(2)}}\right), \quad \text { where } H \in \mathcal{S}(G)
$$

Lemma 4.7. If $f_{\Theta^{(2)}}: \mathcal{S}(G) \rightarrow \mathfrak{P}\left(\Theta^{(2)}\right)$ is $\left(\rho_{\Theta^{(2)}}, S\right)$-saturated then the correspondences

$$
H \mapsto W_{n}\left(\boldsymbol{A}_{H}, \boldsymbol{\Theta}_{H}\right)_{\operatorname{proj}} \quad(H \in \mathcal{S}(G))
$$

and

$$
H \mapsto W_{n}\left(\boldsymbol{A}_{H}, \boldsymbol{\Theta}_{H}\right)_{\text {free }} \quad(H \in \mathcal{S}(G))
$$

afford Mackey functors, respectively.

Proof. Recalling [10, Proposition 10.3], we will prove the lemma by showing that

$$
H \mapsto W_{n}\left(\boldsymbol{A}_{H}, \boldsymbol{\Theta}_{H}\right)_{\text {proj }}, W_{n}\left(\boldsymbol{A}_{H}, \boldsymbol{\Theta}_{H}\right)_{\text {free }} \quad(H \in \mathcal{S}(G))
$$


are $w$-Mackey functors. Most of the proof is already given in the proof of Theorem 12.10 of [10]. It suffices to discuss the part concerning the $(H \times\{ \pm 1\})$-sets $\Theta_{H}^{(0)}$, where $H \in \mathcal{S}(G)$.

Let $H$ and $K$ be subgroups of $G$. Given an injective homomorphism $\varphi$ : $H \rightarrow K$, we have the canonical injective homomorphism $\varphi_{ \pm}: H \times\{ \pm 1\} \rightarrow$ $K \times\{ \pm 1\}$ defined by $\varphi_{ \pm}(h, \epsilon)=(\varphi(h), \epsilon)$ for $h \in H$ and $\epsilon \in\{ \pm 1\}$. The sets $\Theta_{H}^{(0)}$ and $\Theta_{K}^{(0)}$ are an $(H \times\{ \pm 1\})$-set and a $(K \times\{ \pm 1\})$-set, respectively, on which the group $\{ \pm 1\}$ acts freely. Let $\psi: \Theta_{H}^{(0)} \rightarrow \Theta_{K}^{(0)}$ be a $\varphi_{ \pm}$-map, i.e.

$$
\psi((h, \epsilon) t)=\varphi_{ \pm}(h, \epsilon) \psi(t)(=(\varphi(h), \epsilon) \psi(t))
$$

for $h \in H, \epsilon \in\{ \pm 1\}$, and $t \in \Theta_{H}^{(0)}$. Let $\varphi$ denote the pair $(\varphi, \psi)$.

An $R[K]$-module $N$ is usually regarded as an $R[K \times\{ \pm 1\}]$-module via $(k, \epsilon) x$ $=\epsilon(k x)$ for $k \in K, \epsilon \in\{ \pm 1\}$, and $x \in N$. For a pair $\boldsymbol{N}=(N, \beta)$ consisting of an $R[K]$-module $N$ and a $(K \times\{ \pm\})$-map $\beta: \Theta_{K}^{(0)} \rightarrow N$, we define $\varphi^{\#} \boldsymbol{N}=$ $\left(\varphi^{\#} N, \psi^{\#} \beta\right.$ ), where $\varphi^{\#} N$ is an $R[H]$-module and $\psi^{\#} \beta: \Theta_{H}^{(0)} \rightarrow \varphi^{\#} N$, so that the underlying $R$-module of $\varphi^{\#} N$ is the same as $N$ but the $H$-action on $\varphi^{\#} N$ is given by $(h, x) \mapsto \varphi(h) x$ for $h \in H$ and $x \in \varphi^{\#} N$, and $\psi^{\#} \beta(t)=\beta(\psi(t))$ for $t \in \Theta_{H}^{(0)}$.

For a pair $\boldsymbol{M}=(M, \alpha)$ consisting of an $R[H]$-module $M$ and an $(H \times\{ \pm 1\})$ map $\alpha: \Theta_{H}^{(0)} \rightarrow M$, we define $\boldsymbol{\varphi}_{\#} \boldsymbol{M}=\left(\varphi_{\#} M, \psi_{\#} \alpha\right)$, where $\varphi_{\#} M$ is an $R[K]$ module and $\psi_{\#} \alpha: \Theta_{K}^{(0)} \rightarrow \varphi_{\#} M$, by $\varphi_{\#} M=R[K] \otimes_{R[H], \varphi} M$ and

$$
\psi_{\#} \alpha(t)=\sum_{\left[g, t^{\prime}\right]}\left\{g \otimes \alpha\left(t^{\prime}\right) \mid\left[g, t^{\prime}\right] \in K \times_{H, \varphi} \Theta_{H}^{(0)} \text { such that } g \psi\left(t^{\prime}\right)=t\right\}
$$

for $t \in \Theta_{K}^{(0)}$.

These $\boldsymbol{\varphi}^{\#} \boldsymbol{N}$ and $\boldsymbol{\varphi}_{\#} \boldsymbol{M}$ are simple analogies of those in [10, p. 2347]. Thus the conclusion of the lemma above follows from the same arguments used in the proof of Theorem 12.10 of [10].

Let $\rho: \Theta \rightarrow \mathcal{S}(G)$ be a $G$-map and $f: \mathcal{S}(G) \rightarrow \mathfrak{P}(\Theta) ; H \mapsto \Theta_{H}$, an intersection preserving, $\rho$-compatible $G$-map such that $f(G)=\Theta$. Let $\gamma: \Theta^{(2)} \rightarrow \Theta$ be a $G$-map such that the diagram (4.2) commutes and

$$
\gamma\left(\Theta_{H}^{(2)}\right)=\Theta_{H} \quad(H \in \mathcal{S}(G)) .
$$

Lemma 4.8. If $\rho: \Theta \rightarrow \mathcal{S}(G)$ is $S$-bijective, $f: \mathcal{S}(G) \rightarrow \mathfrak{P}(\Theta)$ is $(\rho, S)$-saturated and $f_{\Theta^{(2)}}: \mathcal{S}(G) \rightarrow \mathfrak{P}\left(\Theta^{(2)}\right)$ is $\left(\rho^{(2)}, S\right)$-saturated, then the correspondence

$$
H \mapsto W_{n}\left(\boldsymbol{A}_{H}, \boldsymbol{\Theta}_{H}\right)_{\text {free }} \quad(H \in \mathcal{S}(G))
$$


is a module over the Green functor

$$
H \mapsto \mathrm{SGW}_{0}\left(R, H, S_{H}, \Theta_{H}\right) \quad(H \in \mathcal{S}(G)) .
$$

Proof. We can argue in the same way as in the proof of [10, Theorem 12.10] with a modification using Lemma 4.6.

\section{§5. A deleting-inserting theorem}

Deleting (resp. inserting) $G$-fixed submanifolds from (resp. to) given ambient $G$ manifolds is useful for the study of fixed point data of $G$-manifolds. For example, it has been applied to the study of the Smith problem on tangential representations at fixed points on spheres. In this section we prove Theorem 5.1 below. Let $\mathcal{G}_{p}^{1}(G)$ denote the set of all subgroups $H$ of $G$ possessing normal subgroups $P \unlhd H$ such that $P$ has $p$-power order and $H / P$ is cyclic, where $P$ is possibly the trivial group. An element $H$ of $\mathcal{G}_{p}^{1}(G)$ is called a mod-P $\mathcal{P}_{p}$ cyclic group. We set

$$
\mathcal{G}^{1}(G)=\bigcup_{p \text { prime }} \mathcal{G}_{p}^{1}(G) .
$$

If $H$ lies in $\mathcal{G}^{1}(G)$ then $H$ is referred to as a mod-P cyclic group.

Theorem 5.1 (Deleting-inserting theorem). Let $G$ be a finite Oliver group and $Y$ a smooth $G$-manifold such that the underlying manifold of $Y$ is diffeomorphic to the disk of dimension $n \geq 5$ and $Y^{G} \neq \emptyset$. Let $F_{1}, \ldots, F_{t}$ denote all the underlying spaces of connected components of $Y^{G}$, and let $n_{1}, \ldots, n_{t}$ be non-negative integers. Suppose the following:

(5.1.1) $Y$ satisfies the weak gap condition on $\mathcal{P H}(G)$.

(5.1.2) $\operatorname{dim} Y^{=H} \geq 3$ for any $H \in \mathcal{G}^{1}(G)$.

(5.1.3) $\operatorname{dim} Y^{P} \geq 5$ for any $P \in \mathcal{P}(G)$.

(5.1.4) $\pi_{1}\left(Y^{P}\right)$ is finite and of order prime to $|P|$ for any $P \in \mathcal{P}(G)$.

(5.1.5) For $1 \leq i, j \leq t, n_{i}$ coincides with $n_{j}$ if some connected component $Y_{\alpha}^{H}$ of $Y^{H}, H \in \mathcal{L}(G)$, contains both $F_{i}$ and $F_{j}$.

(5.1.6) For $1 \leq i \leq t, n_{i}$ is equal to 1 if some connected component $Y_{\alpha}^{H}$ of $Y^{H}$, $H \in \mathcal{L}(G)$, contains $F_{i}$ and $\partial Y_{\alpha}^{H} \neq \emptyset$.

(5.1.7) If $\operatorname{dim} Y^{P}=2 \operatorname{dim} Y^{H}$ for $(P, H) \in \mathcal{P H}(G)$ then $(P, H) \in \mathcal{P H}_{2}(G)$ and $\operatorname{dim} Y^{>H} \leq \operatorname{dim} Y^{H}-2$.

Then there exists a smooth $G$-action on the disk $D$ of dimension $n$ such that

(i) $\partial D$ is G-diffeomorphic to $\partial Y$, 
(ii) $D^{G}$ has the form of the disjoint union of copies of $F_{i}$ 's:

$$
D^{G}=\coprod_{i=1}^{t} \coprod_{j=1}^{n_{i}} F_{i, j} \quad\left(\text { each } F_{i, j} \text { is diffeomorphic to } F_{i}\right) \text {, and }
$$

(iii) the normal bundle $\nu\left(F_{i, j}, D\right)$ is $G$-isomorphic to $\nu\left(F_{i}, Y\right)$.

Furthermore if $Y^{H}$ (resp. $\left.Y^{P}\right)$ is connected (resp. simply connected) for an element $H \in \mathcal{G}^{1}(G)$ (resp. $P \in \mathcal{P}(G)$ ), then one can choose the $G$-action so that $D^{H}$ (resp. $D^{P}$ ) is connected (resp. simply connected) for the subgroup $H$ (resp. $P$ ).

Proof. The procedure is the same as that of proving Theorem 1.3 of $[11, \S 5]$. Let $f=(f, b), f:(X, \partial X) \rightarrow(Y, \partial Y)$ and $b: T(X) \oplus \varepsilon_{X}\left(\mathbb{R}^{u}\right) \rightarrow f^{*} T(Y) \oplus \varepsilon_{X}\left(\mathbb{R}^{u}\right)$, be the degree-one $G$-framed map obtained in Section 4 of [11]. Note that for $P \in \mathcal{P}(G), Y^{P}$ is orientable and the map $f^{P}:\left(X^{P}, \partial X^{P}\right) \rightarrow\left(Y^{P}, \partial Y^{P}\right)$ has degree one.

The details of the proof differ in some points from the proof of Theorem 1.3 of $[11, \S 5]$. The differences occur in Steps A and B below.

Step A. The step converting $f^{P}: X^{P} \rightarrow Y^{P}$ to a mod $p$ homology equivalence, where $P \in \mathcal{P}(G)$ possesses $H \in \mathcal{S}(G)$ such that $2 \operatorname{dim} X^{H}=\operatorname{dim} X^{P}$ and $p$ is the prime dividing $|P|$.

Step B. The step converting $f: X \rightarrow Y$ to a homotopy equivalence, when there is (at least one) $H \in \mathcal{S}(G)$ such that $2 \operatorname{dim} X^{H}=\operatorname{dim} X$.

In these steps, the condition (5.1.7) is used to get rid of technical difficulties.

Step $A$. In this step, we set $n_{P}=\operatorname{dim} X^{P}, k_{P}=n_{P} / 2, \lambda=(-1)^{k_{P}}, T=$ $N_{G}(P) / P, w=w_{X^{P}}: T \rightarrow\{ \pm 1\}$, and furthermore

$$
\begin{aligned}
& R=\mathbb{Z}_{(p)}, \\
& S=\left\{g \in T(2) \mid \operatorname{dim}\left(X^{P}\right)^{g}=k_{P}\right\}\left(=S\left(X^{P}\right)\right), \\
& Q=\left\{g \in T(2) \mid \operatorname{dim}\left(X^{P}\right)^{g}=k_{P}-1\right\}\left(=Q\left(X^{P}\right)\right), \\
& \mathfrak{S}=\left\{\left(X^{P}\right)^{g} \mid g \in S\right\}\left(=\mathfrak{S}\left(X^{P}\right)\right), \\
& \Theta^{(0)}=\Theta^{(0)}\left(X^{P}\right), \quad \Theta^{(2)}=\Theta^{(2)}\left(X^{P}\right), \\
& \rho=\rho_{X^{P}}^{(2)}: \Theta^{(2)} \rightarrow \mathcal{S}(T), \quad \Theta=\left(\Theta^{(0)}, \Theta^{(2)}, p_{\Theta^{(0)}}, \rho\right),
\end{aligned}
$$

where $p_{\Theta^{(0)}}: \Theta^{(0)} \rightarrow \Theta^{(2)}$ is the canonical map. Without any loss of generality

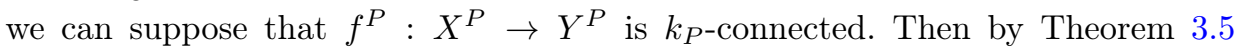
the $T$-surgery obstruction $\sigma\left(f^{P}, b^{P}\right)$ to the $(T, R)$-surgery map $\left(f^{P}, b^{P}\right)$ being a $\mathbb{Z}_{(p)}$-homology equivalence lies in $W_{n_{P}}(R, T, S, Q, \boldsymbol{\Theta})_{\text {free }}$. 
For a subgroup $K$ of $T$, set $S_{K}=S\left(\operatorname{res}_{K}^{T} X^{P}\right), Q_{K}=Q\left(\operatorname{res}_{K}^{T} X^{P}\right), \mathfrak{S}_{K}=$ $\mathfrak{S}\left(\operatorname{res}_{K}^{T} X^{P}\right), \Theta_{K}^{(0)}=\Theta^{(0)}\left(\operatorname{res}_{K}^{T} X^{P}\right), \Theta_{K}^{(2)}=\Theta^{(2)}\left(\operatorname{res}_{K}^{T} X^{P}\right), \rho_{K}=\rho_{\operatorname{res}_{K}^{T} X^{P}}^{(2)}:$ $\Theta_{K}^{(2)} \rightarrow \mathcal{S}(K)$, and

$$
\boldsymbol{\Theta}_{K}=\left(\Theta_{K}^{(0)}, \Theta_{K}^{(2)}, p_{\Theta_{K}^{(0)}}, \rho_{K}\right) .
$$

By Lemmas 2.4, 4.7 and 4.8, the correspondence

$$
K \mapsto W_{n_{P}}\left(R, K, S_{K}, Q_{K}, \Theta_{K}\right)_{\text {free }} \quad(K \in \mathcal{S}(T))
$$

affords a Mackey functor, and moreover a module over the Green functor

$$
K \mapsto \mathrm{SGW}_{0}\left(\mathbb{Z}, K, S_{K}, \Theta_{K}^{(2)} / S_{K}\right) \quad(K \in \mathcal{S}(T))
$$

Thus the argument in $[11, \S 5$, Case 2] using the relation between the equivariant connected sum operation and the $\Omega(T)$-action on the surgery obstruction group (cf. $[11,(5.2)])$, works in the present situation. This ensures that by using equivariant connected sum and $G$-surgery of isotropy type $(P)$, we can convert $f^{P}: X^{P} \rightarrow Y^{P}$ to a $\mathbb{Z}_{(p)}$-homology equivalence.

Step B. In this case, $Y$ is 1 -connected and $n=\operatorname{dim} Y=\operatorname{dim} X$. We set $k=n / 2$, $\lambda=(-1)^{k}, w=w_{X}: G \rightarrow\{ \pm 1\}, R=\mathbb{Z}, S=S(X), Q=Q(X), \mathfrak{S}=\mathfrak{S}(X)$, $\Theta^{(0)}=\Theta^{(0)}(X), \Theta^{(2)}=\Theta^{(2)}(X), \rho=\rho_{X}^{(2)}: \Theta^{(2)} \rightarrow \mathcal{S}(G)$, and

$$
\Theta=\left(\Theta^{(0)}, \Theta^{(2)}, p_{\Theta(0)}, \rho\right),
$$

where $p_{\Theta(0)}: \Theta^{(0)} \rightarrow \Theta^{(2)}$ is the canonical map. Without loss of generality we can suppose that $f: X \rightarrow Y$ is $k$-connected. Since

$$
K_{k}(f ; R)=\operatorname{Ker}\left[f_{*}: H_{k}(X ; R) \rightarrow H_{k}(Y ; R)\right]
$$

is a projective $R[G]$-module but not necessarily a stably free $R[G]$-module, Theorem 6.3 in [2] says that the $G$-surgery obstruction $\sigma(f, b)$ to the $(G, R)$ surgery map $(f, b)$ being a homotopy equivalence lies in the obstruction group $W_{n}(R, G, S, Q, \Theta)_{\text {proj. }}$ But by employing the relation

$$
\left(1+(-\beta)^{\%}\right) \widetilde{K}_{0}(R[G])=0
$$

described in [11, $\S 5$, Case 3] and by taking a suitable equivariant connected sum, we may assume that $K_{k}(f ; R)$ is a stably free $R[G]$-module. Then $\sigma(f, b)$ lies in the obstruction group $W_{n}(R, G, S, Q, \Theta)_{\text {free }}$.

For a subgroup $K$ of $G$, set $S_{K}=S\left(\operatorname{res}_{K}^{G} X\right), Q_{K}=Q\left(\operatorname{res}_{K}^{G} X\right), \mathfrak{S}_{K}=$ $\mathfrak{S}\left(\operatorname{res}_{K}^{G} X\right), \Theta_{K}^{(0)}=\Theta^{(0)}\left(\operatorname{res}_{K}^{G} X\right), \Theta_{K}^{(2)}=\Theta^{(2)}\left(\operatorname{res}_{K}^{G} X\right), \rho_{K}=\rho_{\operatorname{res}_{K}^{G} X}^{(2)}: \Theta_{K}^{(2)} \rightarrow$ $\mathcal{S}(K)$, and

$$
\boldsymbol{\Theta}_{K}=\left(\Theta_{K}^{(0)}, \Theta_{K}^{(2)}, p_{\Theta_{K}^{(0)}}, \rho_{K}\right)
$$


By Lemmas 2.4, 4.7 and 4.8, the correspondence

$$
K \mapsto W_{n}\left(R, K, S_{K}, Q_{K}, \boldsymbol{\Theta}_{K}\right)_{\text {free }} \quad(K \in \mathcal{S}(G))
$$

affords a Mackey functor, and a module over the Green functor

$$
K \mapsto \mathrm{SGW}_{0}\left(\mathbb{Z}, K, S_{K}, \Theta_{K}^{(2)} / S_{K}\right) \quad(K \in \mathcal{S}(G)) .
$$

Thus the argument in [11, §5, Case 3] works in the present situation. Hence, by using equivariant connected sum and $G$-surgery of isotropy type $(\{e\})$, we can convert $f: X \rightarrow Y$ to a homotopy equivalence.

Putting all this together, we have proved the theorem above.

\section{§6. Applications of the deleting-inserting theorem}

Let $G$ be a finite group. One may conjecture that if $V$ and $W$ are $\mathcal{P}$-matched $\mathcal{L}$-free real $G$-modules then $V$ and $W$ are stably Smith equivalent, with which the following is concerned.

Definition 6.1. We call a real $G$-module $V$ admissible if it satisfies the following conditions.

(6.1.1) $V$ satisfies the weak gap condition on $\mathcal{P} \mathcal{H}(G)$.

(6.1.2) $\operatorname{dim} V^{=H} \geq 3$ for any $H \in \mathcal{G}^{1}(G)$.

(6.1.3) $\operatorname{dim} V^{P} \geq 5$ for any $P \in \mathcal{P}(G)$.

(6.1.4) If $\operatorname{dim} V^{P}=2 \operatorname{dim} V^{H}$ for $(P, H) \in \mathcal{P H}(G)$ then $(P, H)$ belongs to $\mathcal{P H}_{2}(G)$ and $\operatorname{dim} V^{>H} \leq \operatorname{dim} V^{H}-2$.

The next lemma is an elaboration of [6, Theorem B]. In [6], we worked with real $G$-modules $V$ such that all transformations $g: V^{H} \rightarrow V^{g H g^{-1}}$ are orientation preserving for $g \in G$ and $H \in \mathcal{S}(G)$ (cf. [6, p. 491 (3.3.6)]).

Lemma 6.2. Let $G$ be an Oliver group, $m$ a positive integer, and $V$ an admissible real $G$-module. Then there exists a smooth $G$-action on the standard sphere $S_{V}$ such that $S_{V}^{G}$ consists of $m$ points $x_{1}, \ldots, x_{m}$ and each $T_{x_{i}}\left(S_{V}\right), 1 \leq i \leq m$, is isomorphic to $V$.

Proof. Let $Y$ be the unit disk $D(V)$ of $V$ with respect to some $G$-invariant inner product. Then $Y$ satisfies the conditions (5.1.1)-(5.1.7). By Theorem 5.1, we obtain a smooth $G$-action on a disk $D_{0}$ such that $D_{0}$ does not have $G$-fixed points and $\partial D_{0}$ is $G$-diffeomorphic to $S(V)=\partial D(V)$. On the other hand, by Theorem 5.1 there exists a smooth $G$-action on a disk $D_{m}$ such that $D_{m}^{G}$ consists of $m$ points $x_{1}, \ldots, x_{m}, \partial D_{0}$ is $G$-diffeomorphic to $S(V)=\partial D(V)$, and the tangential 
representations $T_{x_{i}}\left(D_{m}\right)$ are all isomorphic to $V$. Then glue $D_{0}$ and $D_{m}$ along the boundary and obtain a smooth $G$-action on a homotopy sphere $\Sigma_{V}$ such that $\Sigma_{V}^{G}$ consists of $m$ points $x_{1}, \ldots, x_{m}$ and $T_{x_{i}}\left(\Sigma_{V}\right)$ are isomorphic to $V$. Taking the equivariant connected sum of copies of $\Sigma_{V}$ (cf. [7, Proposition 1.3, Example 1.2]), we can obtain a smooth $G$-action on the standard sphere as desired.

Lemma 1.1 implies that $\mathbb{R}[G]_{\mathcal{L}}^{\oplus 3}$ is an admissible real $G$-module. Hence Theorems 1.3 and 1.4 immediately follow from the lemma above.

Theorem 6.3. Let $G$ be an Oliver group. Let $V_{1}, \ldots, V_{m}$ be $\mathcal{L}$-free real $G$-modules any two of which are $\mathcal{P}$-matched. Then there exists an integer $N_{1}$ such that for any integer $\ell \geq N_{1}$, there exists a smooth $G$-action on the disk $D$ with exactly $m G$-fixed points $x_{1}, \ldots, x_{m}$ for which the tangential representation $T_{x_{i}}(D)$ is isomorphic to $V_{i} \oplus \mathbb{R}[G]_{\mathcal{L}}^{\oplus \ell}$ for $1 \leq i \leq m$.

Proof. Consider the space $F=\left\{x_{1}, \ldots, x_{m}\right\}$ with the trivial $G$-action. We have the $\mathcal{L}$-free real $G$-vector bundle $\nu=\varepsilon_{\left\{x_{1}\right\}}\left(V_{1}\right) \amalg \cdots \amalg \varepsilon_{\left\{x_{m}\right\}}\left(V_{m}\right)$ over $F$. Clearly $\operatorname{res}_{\{e\}}^{G} \nu$ is isomorphic to $\varepsilon_{F}\left(\mathbb{R}^{n}\right)$ for $n=\operatorname{dim} V_{1}$ and $\operatorname{res}_{P}^{G} \nu$ is isomorphic to $\varepsilon_{F}\left(\operatorname{res}_{P}^{G} V_{1}\right)$ for any $P \in \mathcal{P}(G)$. By [14, Theorem 21], there exists an integer $N_{1}$ as desired.

Proof of Theorem 1.5. Let $N_{1}$ be the non-negative integer obtained in Theorem 6.3 for the $G$-modules $V_{1}, \ldots, V_{m}$. There exists an integer $N \geq N_{1}$ such that the real $G$-modules $V_{i} \oplus \mathbb{R}[G]_{\mathcal{L}}^{\oplus N}, 1 \leq i \leq m$, are all admissible. Then for all $\ell \geq N$, the real $G$-modules

$$
W_{i}=V_{i} \oplus \mathbb{R}[G]_{\mathcal{L}}^{\oplus \ell}, \quad 1 \leq i \leq m,
$$

are also admissible. Again by Theorem 6.3, there exists a smooth $G$-action on the disk $Y$ such that $Y^{G}=\left\{x_{1}, \ldots, x_{m}\right\}$ and $T_{x_{i}}(Y) \cong W_{i}$ for $1 \leq i \leq m$. Let $Z$ denote the double $Y \cup_{\partial Y} Y$ of $Y$. Then $Z$ is a sphere having the $G$-fixed points $x_{1}, \ldots, x_{m}$, $x_{1}^{\prime}, \ldots, x_{m}^{\prime}$ such that $T_{x_{i}}(Z) \cong T_{x_{i}^{\prime}}(Z) \cong W_{i}$ for $1 \leq i \leq m$. By Lemma 6.2 , there exist smooth $G$-actions on spheres $S_{i}, 1 \leq i \leq m$, such that $S_{i}^{G}=\left\{x_{i}^{\prime \prime}\right\}$ and $T_{x_{i}^{\prime \prime}}\left(S_{i}\right) \cong W_{i}$. Let $S$ denote the $G$-manifold obtained as the $G$-connected sum of $Z$ and $S_{i}, 1 \leq i \leq m$, at pairs $\left(x_{i}^{\prime}, x_{i}^{\prime \prime}\right) \in Z \times S_{i}$. Then the underlying manifold of $S$ is diffeomorphic to the standard sphere and moreover $S$ possesses the properties required in Theorem 1.5.

Let $\mathrm{WP}(G)$ denote the set consisting of $[V]-[W] \in \mathrm{RO}(G)^{\mathcal{L}}$ such that $V$ and $W$ both are $\mathcal{L}$-free and satisfy the weak gap condition on $\mathcal{P} \mathcal{H}_{2}(G)$. Note that $G$ is a weak gap group if and only if $\mathrm{WP}(G)_{\mathcal{P}}=\mathrm{RO}(G)_{\mathcal{P}}^{\mathcal{L}}$. Since the set

$$
-\mathrm{WP}(G)=\{-x \in \operatorname{RO}(G) \mid x \in \mathrm{WP}(G)\}
$$

coincides with $\operatorname{WP}(G)$, we can prove the next proposition without difficulties. 
Proposition 6.4. The set $\mathrm{WP}(G)$ is a subgroup of $\mathrm{RO}(G)$.

Theorem 1.9 can be reformulated as follows:

Theorem 6.5. If $H$ is a subgroup of an Oliver group $G$ then

$$
\operatorname{ind}_{H}^{G}\left(\mathrm{WP}(H)_{\mathcal{P}}\right) \subset \operatorname{Sm}(G)_{\mathcal{P}}^{\mathcal{L}} .
$$

For a pair $(P, H) \in \mathcal{P H}(G)$, define a $\mathbb{Z}$-linear map $f_{P, H}: \mathrm{RO}(G) \rightarrow \mathbb{Z}$ by

$$
f_{P, H}([V])=\operatorname{dim} V^{P}-2 \operatorname{dim} V^{H} .
$$

Next define

$$
\begin{aligned}
& \mathrm{P}_{+}\left(\mathcal{P H}_{2}(G)\right)=\left\{x \in \operatorname{RO}(G)^{\mathcal{L}} \mid f_{P, H}(x) \geq 0 \text { for all }(P, H) \in \mathcal{P H}_{2}(G)\right\}, \\
& \mathrm{P}_{-}\left(\mathcal{P H}_{2}(G)\right)=\left\{x \in \operatorname{RO}(G)^{\mathcal{L}} \mid f_{P, H}(x) \leq 0 \text { for all }(P, H) \in \mathcal{P} \mathcal{H}_{2}(G)\right\} .
\end{aligned}
$$

It is clear that $\mathrm{P}_{-}\left(\mathcal{P H}_{2}(G)\right)=-\mathrm{P}_{+}\left(\mathcal{P H}_{2}(G)\right)$.

Lemma 6.6. For an arbitrary finite group $G$, we have

$$
\mathrm{P}_{+}\left(\mathcal{P H}_{2}(G)\right) \cup \mathrm{P}_{-}\left(\mathcal{P H}_{2}(G)\right) \subset \mathrm{WP}(G) .
$$

Proof. Let $x=[V]-[W] \in \mathrm{P}_{+}\left(\mathcal{P H}_{2}(G)\right)$, where $V$ and $W$ are $\mathcal{L}$-free real $G$ modules. By [13, Proposition 2.3], $W$ is isomorphic to a $G$-submodule of $\mathbb{R}[G]_{\mathcal{L}}^{\oplus m}$, where $m=\operatorname{dim} W$. Thus we can assume $W=\mathbb{R}[G]_{\mathcal{L}}^{\oplus m}$ without any loss of generality. Then the inequality

$$
f_{P, H}(x)=\left(\operatorname{dim} V^{P}-2 \operatorname{dim} V^{H}\right)-m\left(\operatorname{dim}\left(\mathbb{R}[G]_{\mathcal{L}}\right)^{P}-2 \operatorname{dim}\left(\mathbb{R}[G]_{\mathcal{L}}\right)^{H}\right) \geq 0
$$

for $(P, H) \in \mathcal{P H}_{2}(G)$ reads

$$
\operatorname{dim} V^{P}-2 \operatorname{dim} V^{H} \geq m\left(\operatorname{dim}\left(\mathbb{R}[G]_{\mathcal{L}}\right)^{P}-2 \operatorname{dim}\left(\mathbb{R}[G]_{\mathcal{L}}\right)^{H}\right) .
$$

Since the right-hand side above is non-negative, $V$ satisfies the weak gap condition on $\mathcal{P H}_{2}(G)$ as also does $W=\mathbb{R}[G]_{\mathcal{L}}^{\oplus m}$, which ensures that the element $x=$ $[V]-[W]$ belongs to $\mathrm{WP}(G)$, hence $\mathrm{P}_{+}\left(\mathcal{P H}_{2}(G)\right) \subset \mathrm{WP}(G)$.

In addition, we have

$$
\mathrm{P}_{-}\left(\mathcal{P H}_{2}(G)\right)=-\mathrm{P}_{+}\left(\mathcal{P \mathcal { H } _ { 2 }}(G)\right) \subset-\mathrm{WP}(G)=\mathrm{WP}(G) .
$$

This completes the proof.

The next claim immediately follows from Theorem 6.5 and Lemma 6.6.

Theorem 6.7. If $H$ is a subgroup of an Oliver group $G$ then

$$
\operatorname{ind}_{H}^{G}\left(\mathrm{P}_{+}\left(\mathcal{P} \mathcal{H}_{2}(H)\right)_{\mathcal{P}} \cup \mathrm{P}_{-}\left(\mathcal{P} \mathcal{H}_{2}(H)\right)_{\mathcal{P}}\right) \subset \operatorname{Sm}(G)_{\mathcal{P}}^{\mathcal{L}} .
$$


Proof of Theorem 1.10. It is clear that

$$
\mathrm{RO}(H)_{\mathcal{H}}^{\mathcal{L}} \subset \mathrm{P}_{+}\left(\mathcal{P} \mathcal{H}_{2}(H)\right)_{\mathcal{H}} \subset \mathrm{P}_{+}\left(\mathcal{P} \mathcal{H}_{2}(H)\right)_{\mathcal{P}}
$$

and

$$
\operatorname{ind}_{H}^{G}\left(\mathrm{RO}(H)_{\mathcal{H}}\right) \subset \operatorname{RO}(G)_{\mathcal{H}} .
$$

Thus Theorem 1.10 follows from Theorem 6.7.

\section{Acknowledgements}

The author wishes to express his deepest gratitude to the referee for helpful comments. This research was partially supported by a Grant-in-Aid for Scientific Research (KAKENHI) No. 225400875.

\section{References}

[1] A. Bak, K-theory of forms, Ann. of Math. Stud. 98, Princeton Univ. Press, Princeton, 1981. Zbl 0465.10013 MR 0632404

[2] A. Bak and M. Morimoto, Equivariant intersection theory and surgery theory for manifolds with middle dimensional singular sets, J. K-Theory 2 (2008), Special Issue 03, 507-600. Zbl 1156.57028 MR 2465449

[3] S. E. Cappell and J. L. Shaneson, The codimension two placement problem and homology equivalent manifolds, Ann. of Math. 99 (1974), 277-348. Zbl 0279.57011 MR 0339216

[4] X. M. Ju, The Smith set of the group $S_{5} \times C_{2} \times \cdots \times C_{2}$, Osaka J. Math. 47 (2010), 215-236. Zbl 1228.55004 MR 2666133

[5] X. M. Ju, K. Matsuzaki and M. Morimoto, Mackey and Frobenius structures on odd dimensional surgery obstruction groups, K-Theory 29 (2003), 285-312. Zbl 1044.19003 MR 2029081

[6] E. Laitinen and M. Morimoto, Finite groups with smooth one fixed point actions on spheres, Forum Math. 10 (1998), 479-520. Zbl 0905.57023 MR 1631012

[7] E. Laitinen, M. Morimoto and K. Pawałowski, Deleting-inserting theorem for smooth actions of finite nonsolvable groups on spheres, Comment. Math. Helv. 70 (1995), 10-38. Zbl 0843.57034 MR 1314939

[8] M. Morimoto, Bak groups and equivariant surgery, $K$-Theory 2 (1989), 465-483. Zbl 0669.57018 MR 0990572

[9] , Bak groups and equivariant surgery II, $K$-Theory $\mathbf{3}$ (1990), 505-521. Zbl 0712.57013 MR 1071893

[10] Induction theorems of equivariant surgery obstruction groups, Trans. Amer. Math. Soc. 355 (2003), 2341-2384. Zbl 1019.19002 MR 1973993

[11] _ Fixed-point sets of smooth actions on spheres, J. K-Theory 1 (2008), 95-128. Zbl 1154.57037 MR 2424568

[12] M. Morimoto and K. Iizuka, Extendibility of $G$-maps to pseudoequivalences to finite $G$ CW-complexes whose fundamental groups are finite, Osaka J. Math. 21 (1984), 59-69. Zbl 0542.57031 MR 0736970

[13] M. Morimoto and K. Pawałowski, The equivariant bundle subtraction theorem and its applications, Fund. Math. 161 (1999), 279-303. Zbl 0947.57035 MR 1716021 
[14] _ Smooth actions of finite Oliver groups on spheres, Topology 42 (2003), 395-421. Zbl 1019.57020 MR 1941442

[15] B. Oliver, Fixed point sets and tangent bundles of actions on disks and euclidean spaces, Topology 35 (1996), 583-615. Zbl 0861.57047 MR 1396768

[16] _ Fixed-point sets of groups on finite acyclic complexes, Comment. Math. Helv. 50 (1975), 155-177. Zbl 0304.57020 MR 0375361

[17] K. Pawałowski and R. Solomon, Smith equivalence and finite Oliver groups with Laitinen number 0 or 1, Algebr. Geom. Topol. 2 (2002), 843-895. Zbl 1022.57019 MR 1936973

[18] C. T. C. Wall, Surgery on compact manifolds, Academic Press, London, 1970. Zbl 0219.57024 MR 0431216 